\title{
A Proposed Methodology for Software Engineering of Agent Systems
}

\section{Asmaa Yaseen Hammo}

asmahammo@uomosul.edu.iq

College of Computer Sciences and

Mathematics/University of Mosul

\section{Maher Talal Alasaady}

maher.alasaady@ntu.edu.iq

Northen Technical University

\section{Received 2011/05/11}

\begin{abstract}
In recent years, computer systems that make use of software agents are increased, due to the increased size and complexity of these systems, as well as, because the benefits provided by agents to facilitating the developing process and requirements representation for these systems. Traditional software engineering methodologies are not enough to be used in the development of such systems, because the special characteristics for software agent, therefore there are many proposed Agent Based Software Engineering (ABSE) methodologies to support software engineers in the agents based systems development, However, some of them were designed without adhering to all main stages of software development life cycle, as well as they are lacking for representation of most agents features, additionally, most of them are not represents the implementation phase or represented it but in unclear and unpractical manner.
\end{abstract}

To address these issues, this research is investigated the existing methodologies and diagnose its strengths and weaknesses, then construct a proposed ABSE methodology depending on combining of strengths for existing methodologies, and delete or modify its weaknesses. This methodology is covered all main stages of software development life cycle, and dealing with problems in the existing methodologies. The requirements phase and part of the design phase were rely on Tropos methodology, whereas the implementation phase and part of the analysis and design phases were rely on MASD methodology, these stages has been modified by deleting its weaknesses and add some other novel techniques.

To improve the new proposed methodology, a running agent based e-commerce system has been developed depending on stages of this methodology, and implement it with Java programming language and XML using the Jadex platform Keywords: MASD, Agent system, Tropos, AMART, Jadex.

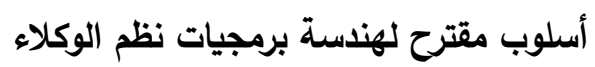

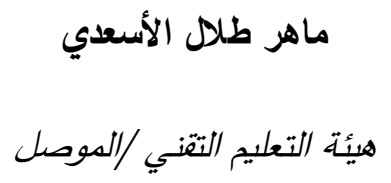

تاريخ قبول البحث: 2011/06/21

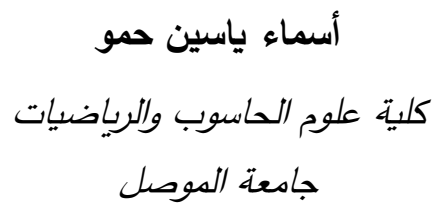

تاريخ استلام البحث: 2011/05/11 


\section{الملخص}

تزايدت في السنوات الأخيرة النظم الحاسوبية التي تحقق الاستفادة من الوكلاء البرمجية، بسبب زيادة حجم

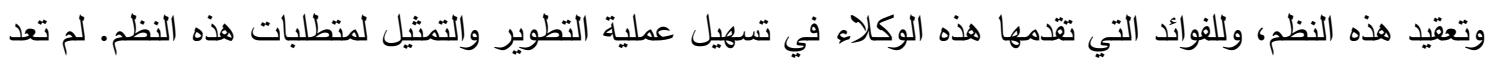
أساليب هندسة البرمجيات التقليدية كافية لاستخدامها في تطوير مثل هذه النظم بسبب المميزات الخاصة للوكيل

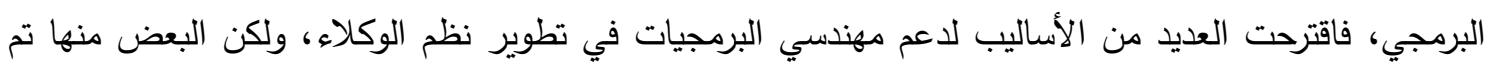

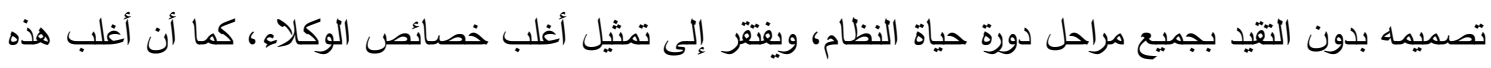

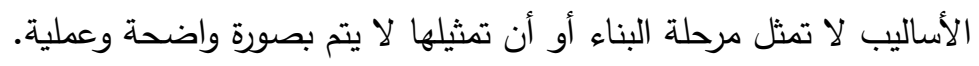

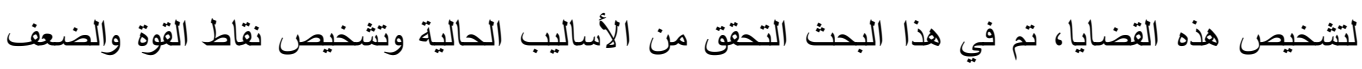

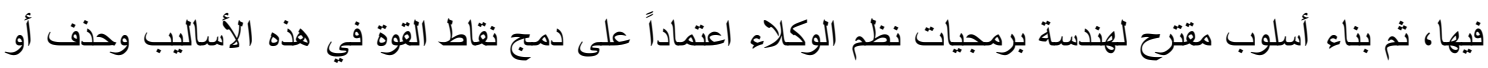
تعديل نقاط الضعف مع إضافة بعض التقنيات الأخرى المستحدثة ليغطي هذا الأسلوب جميع مراحل دورة حياة النظام ومعالجة المشاكل الموجودة في الأساليب الحالية.

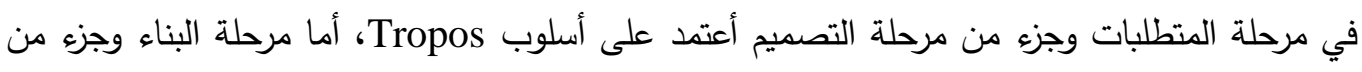

مرحلة التحليل والتصميم فأعتمد على أسلوب MASD، وتم تعديل هذه المراحل بحذف نقاط الضعف وإضافة

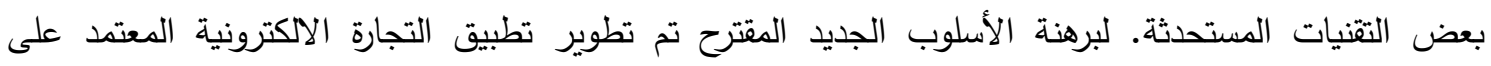

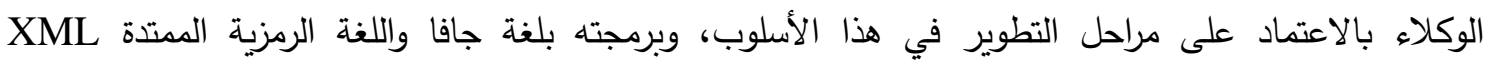

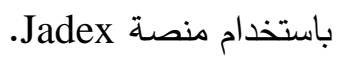
الكلمات المفتاحية: MASD ، نظام الوكيل ، Tadex ،AMART Tropos ،

\section{1- المقدمة:}

الوكيل Agent هو كيان برمجي يمتلك خصـائص ذكية مثل الاستقلالية والتنكير والتتقل والاجتماعية

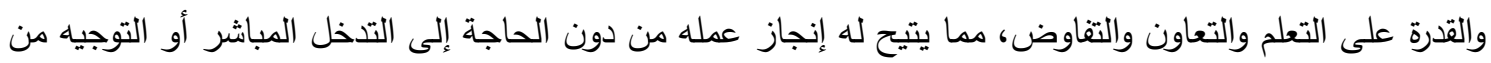

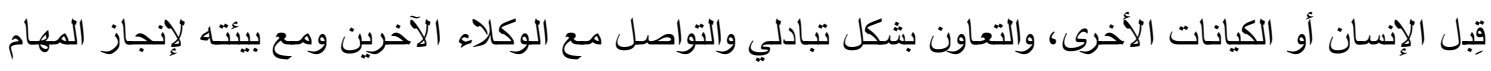

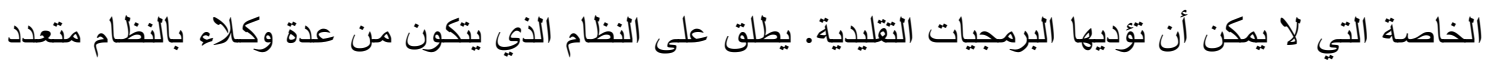
الوكلاء Multi Agent System (MAS)، حيث أن كل وكيل في النظام لديه معلومات و قدرات غير مكتملة لغرض حل المسائل، وبالتالي كل وكيل لديه وجهة نظر محدودة و لا يوجد تحكم عام للنظام وتكون البيانات غير مركزية والمعالجة تكون غير متزامنة [20]. لعملية التواصل بين الوكلاء يتم استخدام لغة تواصل الوكلاء Communication Language (ACL)

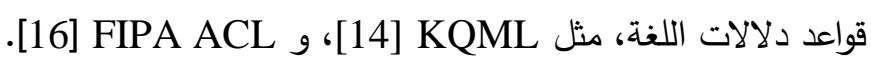
جذبت نظم الوكلاء الكثير من الانتباه خلال السنوات الماضية، وذلك لأنها قدمت أنموذجاً جديداً لتطوير النظم البرمجية، ودعماً لذلك فقد تم اقتراح العديد من أساليب هندسة البرمجيات لتطوير هذه النظم [3 مثل Gaia

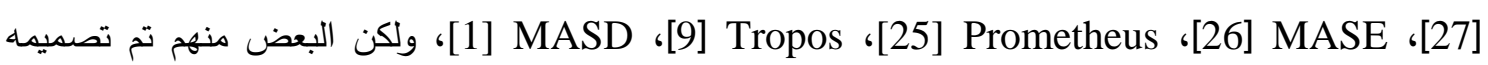
بدون التقيد بجميع مراحل دورة حياة النظام، والبعض الآخر يفتقر إلى تمثيل أغلب خصائص الوكلاء، وأغلب هذه الأساليب لا تمثل مرحلة البناء أو تمثلها بصورة غير واضئ دورة واضحة وغير عملية. 


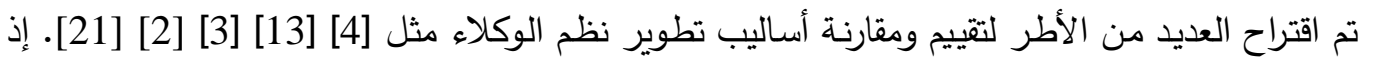

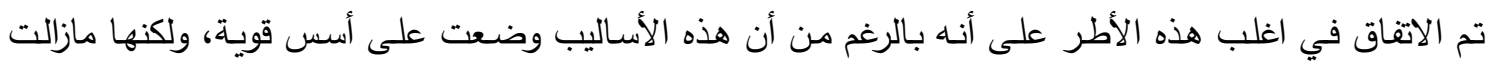

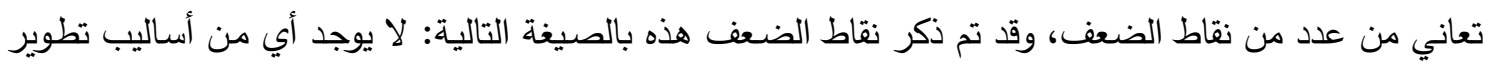
نظم الوكلاء الحالية مقبولاً بوصفه معياراً، ولا يوجد أي واحد منهم يستخدم بطريقة واسعة. حتى الآن لم يتم بناء

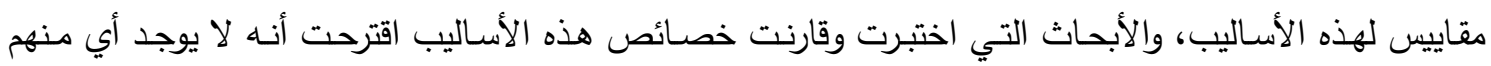

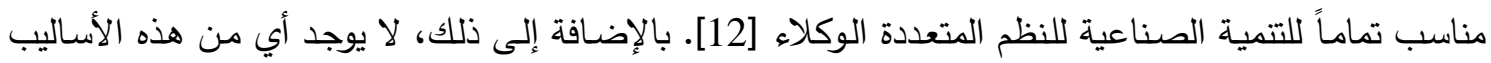

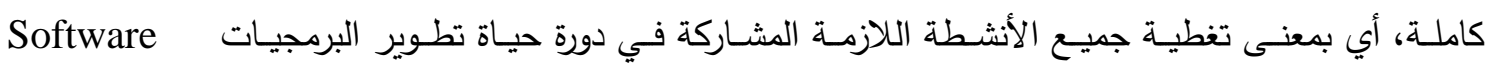
Development Life Cycle (SDLC) لنظم الوكلاء. وعلاوة على ذلك، فإن معظم أساليب تطوير الوكلاء تعاني من فجوة بين نماذج التصميم ولغات

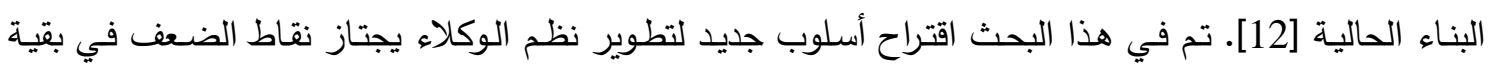
الأساليب ويكون مبنياً على أساس المفاهيم والخصائص والنماذج والناحية العملية والتطبيقية.

\section{2- أهداف البحث}

يهدف البحث إلى تطوير أسلوب جديد لهندسة برمجيات نظم الوكلاء يحمل الصفات التالية:

$$
\begin{aligned}
& \text { 1. يكون شاملاً يغطي جميع مراحل تطوير النظام الأساسية. } \\
& \text { 2. يغطي أغلب خصائص الوكلاء. } \\
& \text { 3. التعامل مع مفهوم الوكيل بدرجة عالية من التجريد. }
\end{aligned}
$$

4. التعامل مع مرحلة المتطلبات بدرجة مفصلة ذلك لأهمية هذه المرحلة لبقية المراحل ولمشاركة الزبون في

$$
\text { هذه المرحلة. }
$$

5eliefs, Foundation of Intelligent Physical Agent (FIPA) وهيكلية التوافق مع مقاييس

.Desires, Intentions (BDI) 6. التعامل مع مرحلة البناء بشكل تفصيلي لغرض تتفيذ النظام بسهولة وتوافق النظام قيد التطوير مع أغلب منصات وأطر تطوير الوكلاء.

BDI 3.

تحتوي وكلاء BDI الاتجاهات العقلانية وهي الحقائق Beliefs والرغبات Desires والنوايا التي يمكن أن توظف لنمذجة قدراتها المعرفية، وأيضاً لتحديد حالتها الداخلية وتزويدها بالتنكير

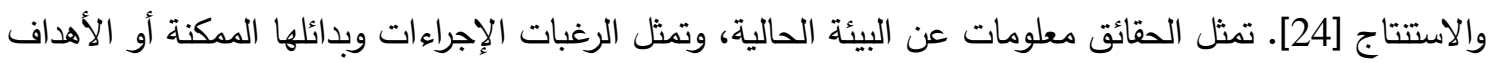

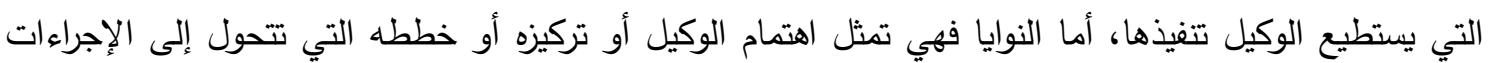
لتنفيذ الأهداف [8].

\section{4- مقاييس FIPA}

منظمة FIPA هي منظمة دولية تكرس جهودها لتعزيز صناعة الوكلاء الذكية من خلال تطوير المقاييس لاعم العمل المشترك بين الوكلاء والتطبيقات المسندة إلى الوكيل. تضم هذه المقاييس بنية الوكلاء وطريقة 
تجريدها، طرق التواصل بين الوكلاء، لغات محتوى الرسائل بين الوكلاء، هيكلية الرسائل، بروتوكولات التفاعل بين

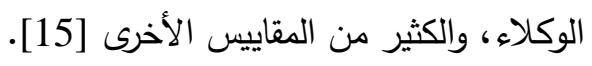
5- أساليب هندسة برمجيات نظم الوكلاء

هندسة برمجيات نظم الوكلاء هي واحدة من أحدث نماذج البرمجة وهي انتقال كبير في هندسة البرمجيات

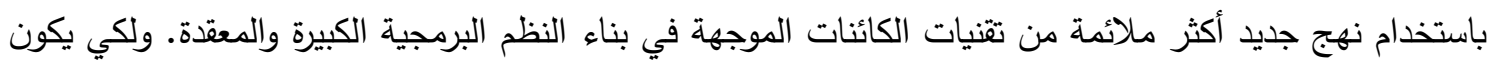
المفهوم قابلاً للتطبيق، هناك حاجة لإتباع نهج موحد في كل المراحل خلال دورة حياة تطوير نظام الوكيل والذي لناءي

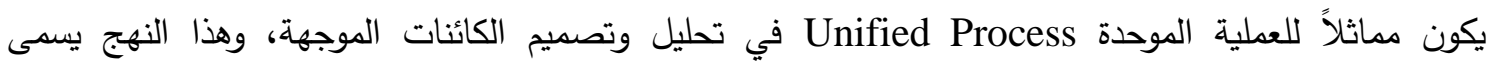
بالأسلوب. يمكن تعريف أسلوب هندسة البرمجيات هو مجموعة منظمة من المبادئ التوجيهية والمفاهيم أو الأنشطة

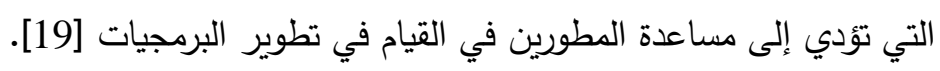

\section{1. أسلوب Tropos}

هو أسلوب لتطوير البرمجيات ويتم فيه استخدام أنموذج الوكيل البرمجي في عملية تطوير النظام. يستتد Tropos

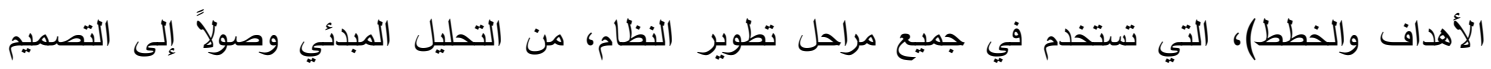

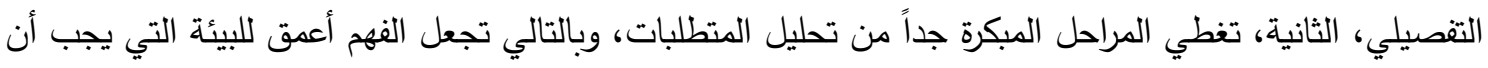
يعمل فيها النظام بالإضافة إلى نوع التفاعلات التي يجب أن تحدث بين الوكلاء [23]. يحتوي الأسلوب على أربع

مراحل رئيسية [9]: أ- المتطلبات المبئية Early Requirements: يتم في هذه المرحلة فهم المشكلة من خلال دراسة للوضع الحالي، وناتج هذه المرحلة هو الأنموذج التتظيمي الذي يشمل الفاعلين وأهداف وتبعيات كل منهم. المتطلبات المبئية تثمل مخططين رئيسيين وهما، مخطط الفاعل ومخطط الهدف، إذ يتكون مخطط الهدف من تنقية مخطط الفاعل من خلال التركيز على أهداف فاعل واحد فقط. ب- - المتطلبات المتقدمة Late Requirements: في هذه المرحلة يوصف النظام ليكون ضمن بيئته التشغيلية جنباً إلى جنب مع صفاته ومهامه. النظام يكون على شكل فاعل واحد لديه عدد من التبعيات مع الفاعلين الآخرين في التظظيم، وهذه التبعيات تحدد منطلبات النظام الوظيفية وغير الوظيفية. التصميم الهيكلي Architectural Design: يتم في هذه المرحلة تعريف هيكلية النظام العامة التي تتكون من النظم الفرعية التي تكون مترابطة من خلال البيانات والتحكم وغيرها من التبعيات، وتتمحور هذه المرهلة

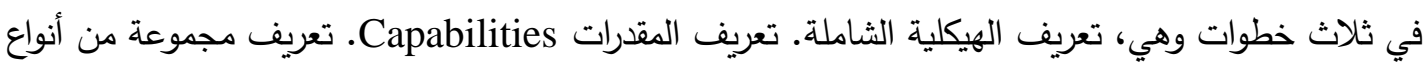
الوكيل Agent Types وتوزيع كل واحد منهم أو أكثر على المقدرات. دـ - التصميم التفصيلي Detailed Design: يتم في هذه المرحلة تعريف سلوك كل عنصر هيكلي على حدة في مزيد من التفاصيل، ويتم تعريف أهداف وحقائق ومقدرات الوكيل بالتفصيل، جنباً إلى جنب مع التفاعل بينهما.

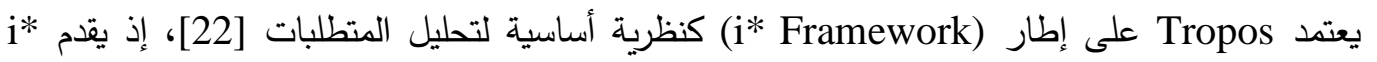
بعض المفاهيم مثل الفاعلين، والأهداف، والتبعيات التي تهذف إلى نمذجة الهياكل الاجتماعية. 
مزايا أسلوب Tropos:

هحتوي على مرحلة الدتطلبات المبدئية والتي تكون بشكل مبسط وتكون مفهومة للمستخدم العادي، حيث

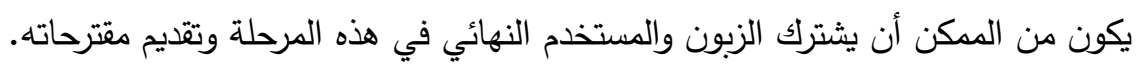

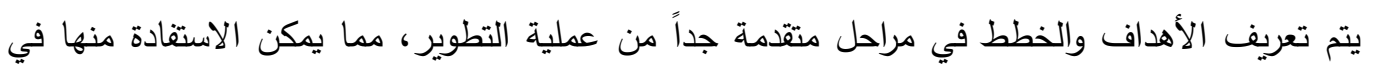
عملية التعديل بصورة مبكرة ولا يؤثر على بقية المراحل. اعتماد الأسلوب على التبعيات بين الفاعلين وبين أهدافهم في النظام، إذ يمكن الاستفادة منها في أولويات

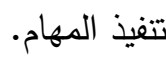
يحتوي على مخطط يمثل النظام الثامل والنظم الفرعية في مرحلة التصميه، وهذا الخخطط يعطي وصفاً شاملاً للنظام الكلي.

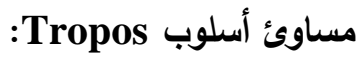

الطريقة المستخدمة في تعريف حقائق الوكيل هي عن طريق الاستتناج المعتدة على النتائج السابقة،

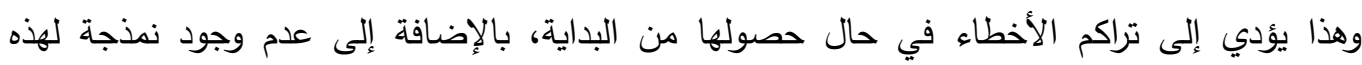
الحقائق. عدم وجود نماذج للأهداف والخطط لتعريفها بشكل واضح في عملية البناء الفعلي للنظام.

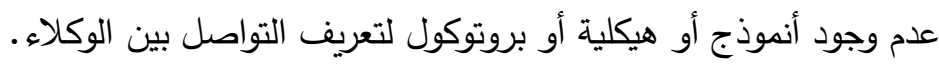

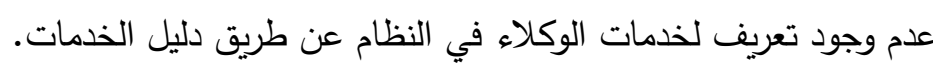
عدم وجود تفصيل لمرحلة البناء وهذا يؤدي إلى فجوة بين مرحلة التصميم التفصيلي وعملية البناء الفعلي لإني اللنظام.

$$
\text { MASD } 2
$$

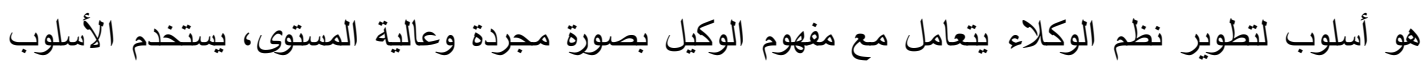

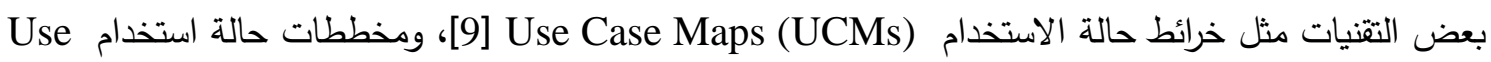
MASD Case Diagrams (UCDs)

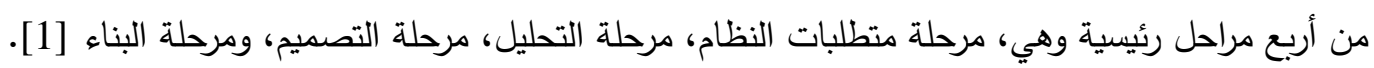

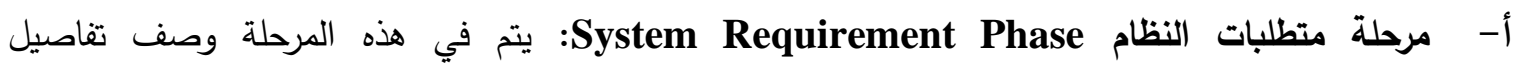

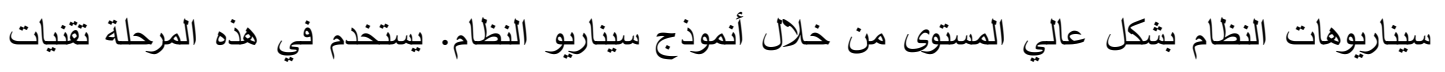

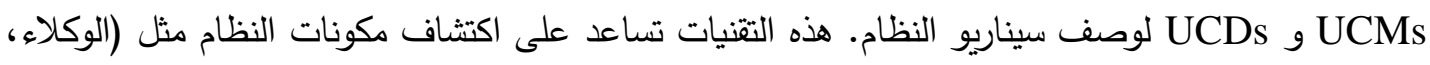

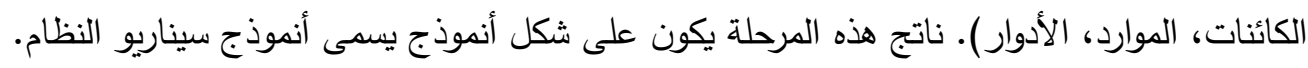

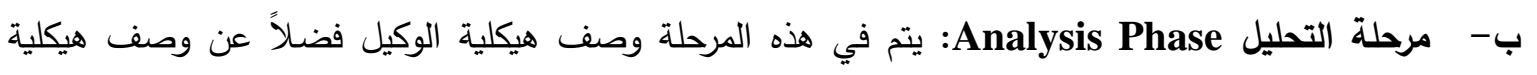

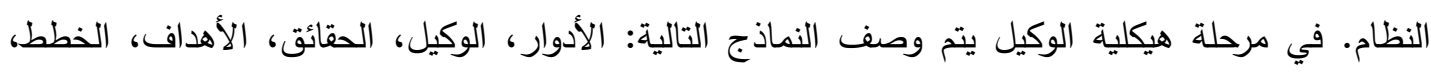

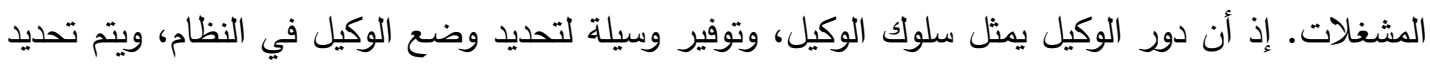

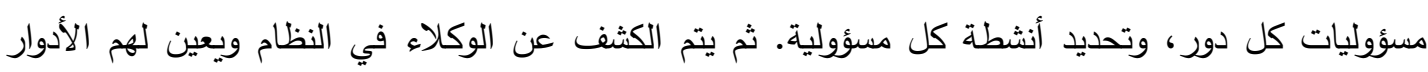


المناسبة. أنموذج الوكيل يتألف من خطوتين. الأولى، تعريف الوكلاء داخل النظام وتتقية الأدوار لتناسب

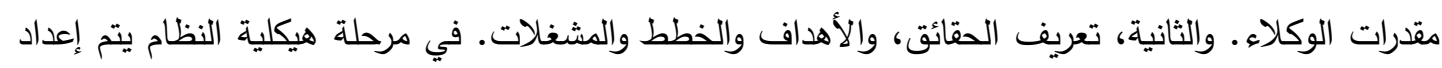
أنموذج لتفاعل الوكلاء مع بعض في النظام باستخدام سيناريوهات UCMs. ويتم إعداد أنموذج علاقات الوكيل ليجسد العلاقات بين الوكلاء، ويتم إعداد أنموذج خدمات الوكيل لكثف الخدمات التي ينبغي أن تتوفر لكل وكيل في النظام.

ج- مرحلة التصميم Design Phase: يتم في هذه المرحلة تمثيل مفصل للنماذج وتحويلها إلى تركيبات التصميم. وهذه التركيبات تكون تمهيداً لبناء النظام، إذ تتم مراجعة النماذج التي تم إعدادها في مرحلة التحليل

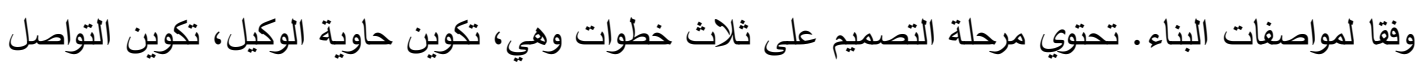
المتبادل بين الوكلاء، وتكوين دليل الخدمات.

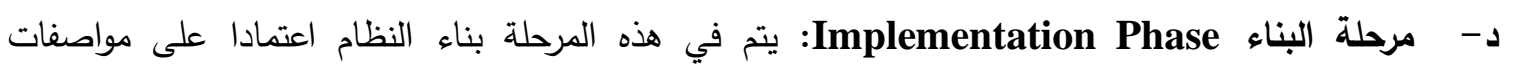

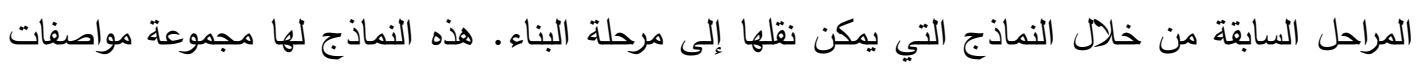
التصميم لتعرض كيفية تنظيم نظام الوكلاء ومكوناته.

مزايا أسلوب MASD:

يحتوي على نماذج لكل من الحقائق والأهداف والخطط والأدوار، وهذه النماذج توضح مكونات النظام وتسهل عملية البناء الفعلي للنظام. يحتوي على أنموذج التواصل التبادلي بين الوكلاء، مما يسهل عملية إعداد صيغ الرسائل المتبادلة بين الوكلاء في النظام. يحتوي على حاوية الوكيل التي تضم مكونات الوكيل مما يسهل عملية البناء الفعلي للنظام. مساوئ أسلوب MASD: مسوب

مرحلة المتطلبات تكون معقدة نسبياً، ولا تأخذ بنظر الاعتبار الزبون الذي سوف يشترك في هذه المرحلة

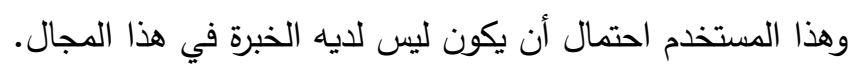

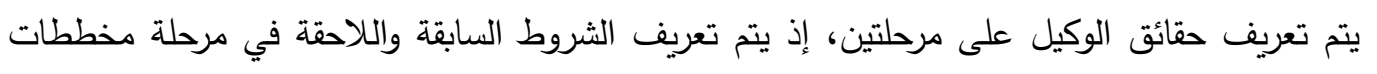

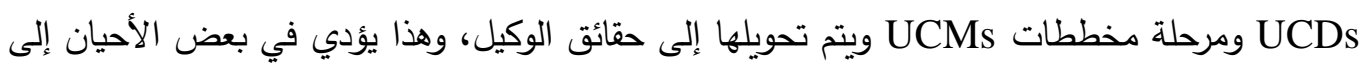

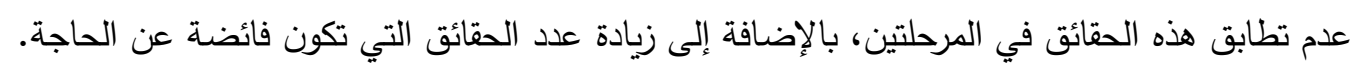

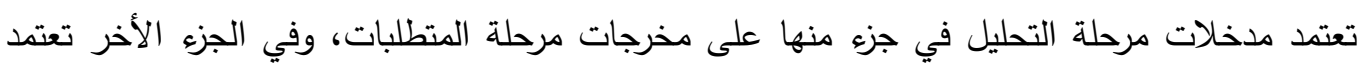

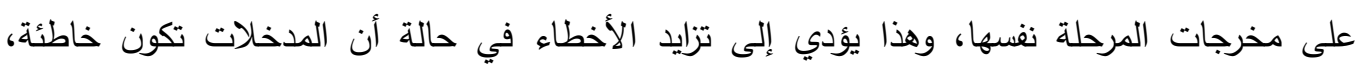

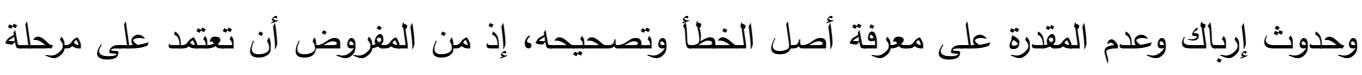
المتطلبات بشكل كلي. يتم تعريف الأهداف والخطط من خلال مراجعة نماذج UCMs و UCDs ومن خلال المهام، ولكن هذه المخططات قابلة للتعديل والمراجعة باستمرار، وهذا سوف يؤدي إلى تغيير الأهداف والخطط في مرحلة فرادي التحليل، إذ من المفروض أن يتم تعريف الأهداف والخطط مباشرة في مرحلة المتطلبات. 


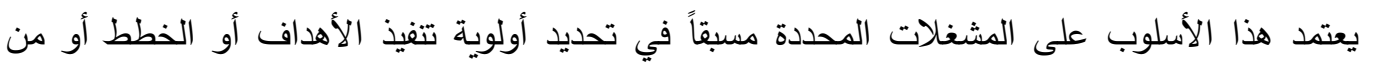

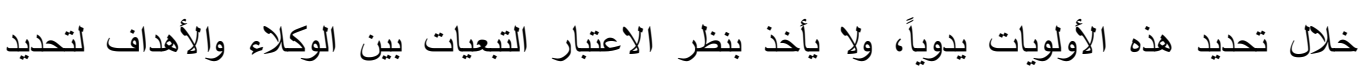

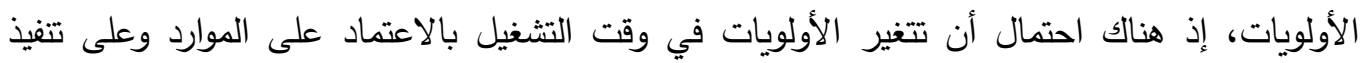
أهداف أو خطط معينة.

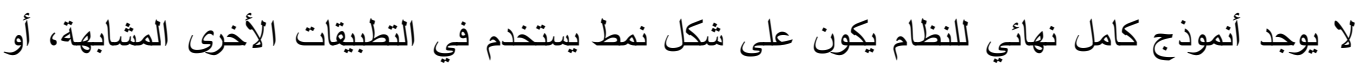
الرجوع إليه في حالة التعديل والتحديث، ففي حالة تعديل المتطلبات يجب تعدئ تعديل كافة النماذج في مرحلة

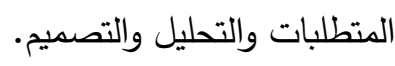
يتم تعريف الخدمات من خلال حالات الاستخام في مخططات UCDs، وهذا يؤدي إلى عدد كبير من التصات

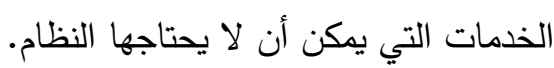

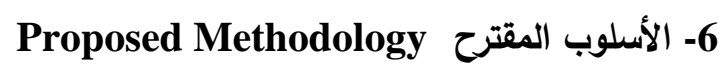
تم تطوير أسلوب هجين من أساليب تطوير الوكلاء الأخرى من خلال دمج التقنيات ذات المزايا القوية

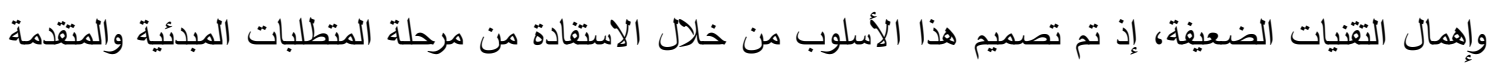

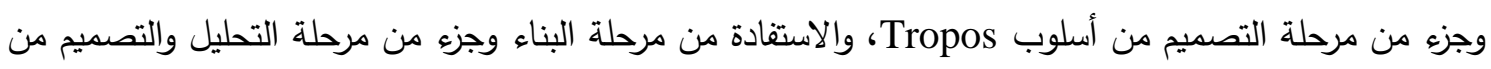

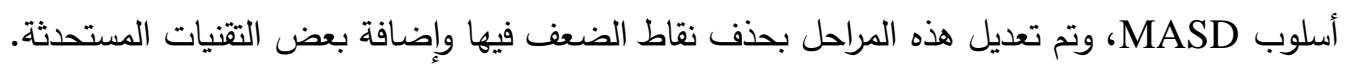

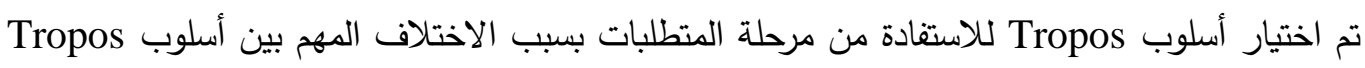

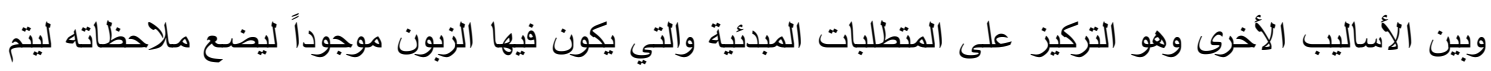

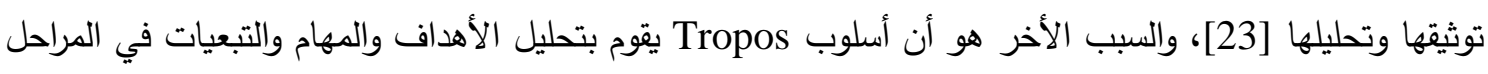

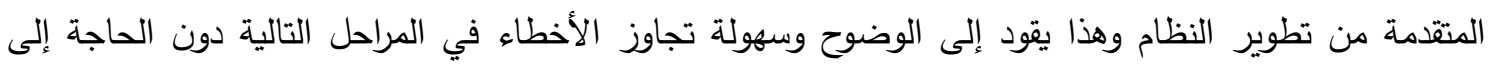

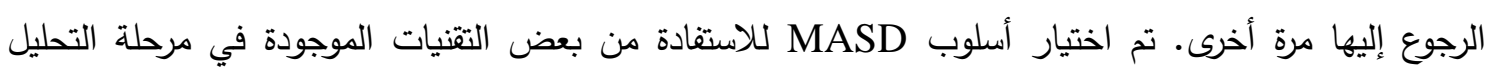

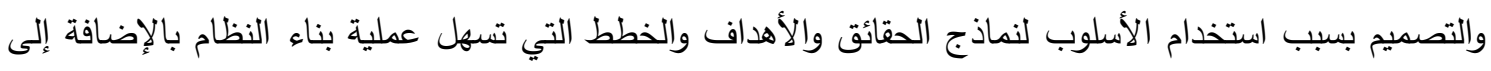

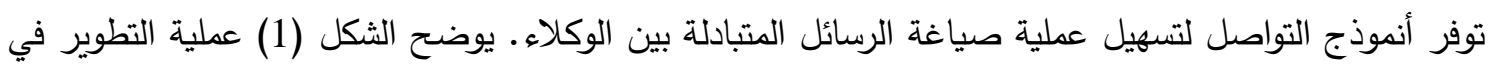

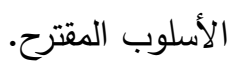

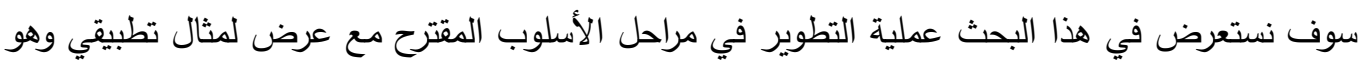
"تطبيق التجارة الاككترونية باستخدام الوكلاء البرمجية" لغرض برضية فرهنة التطبيق العملي لهذا الأسلوب الجديد.

Requirement Phase 1-8 مرحلة المتطلبات

في مرحلة الدتطلبات يتم وصف النظام بتمثيل عالي المستوى لتكوين مخطط الفاعل، ويستخدم هذا

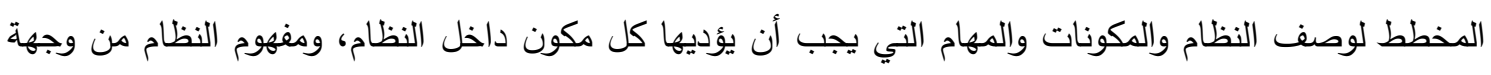

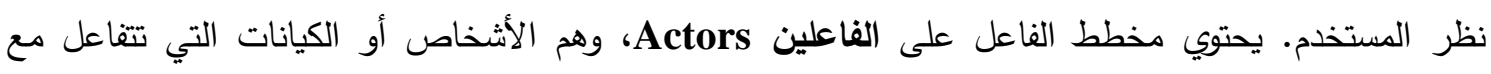

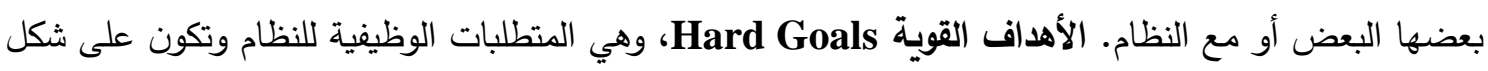

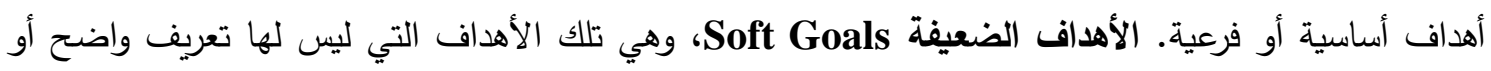

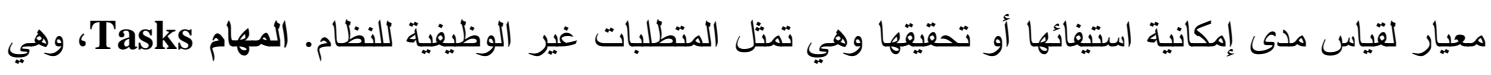

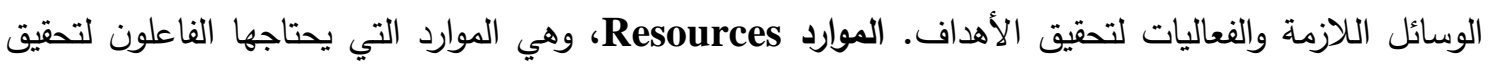


الأهداف. التبعيات Dependences، وهي يمكن أن تُقسَر بأن هناك فاعلاً تابعاً Depender وفاعلاً متبوعاً

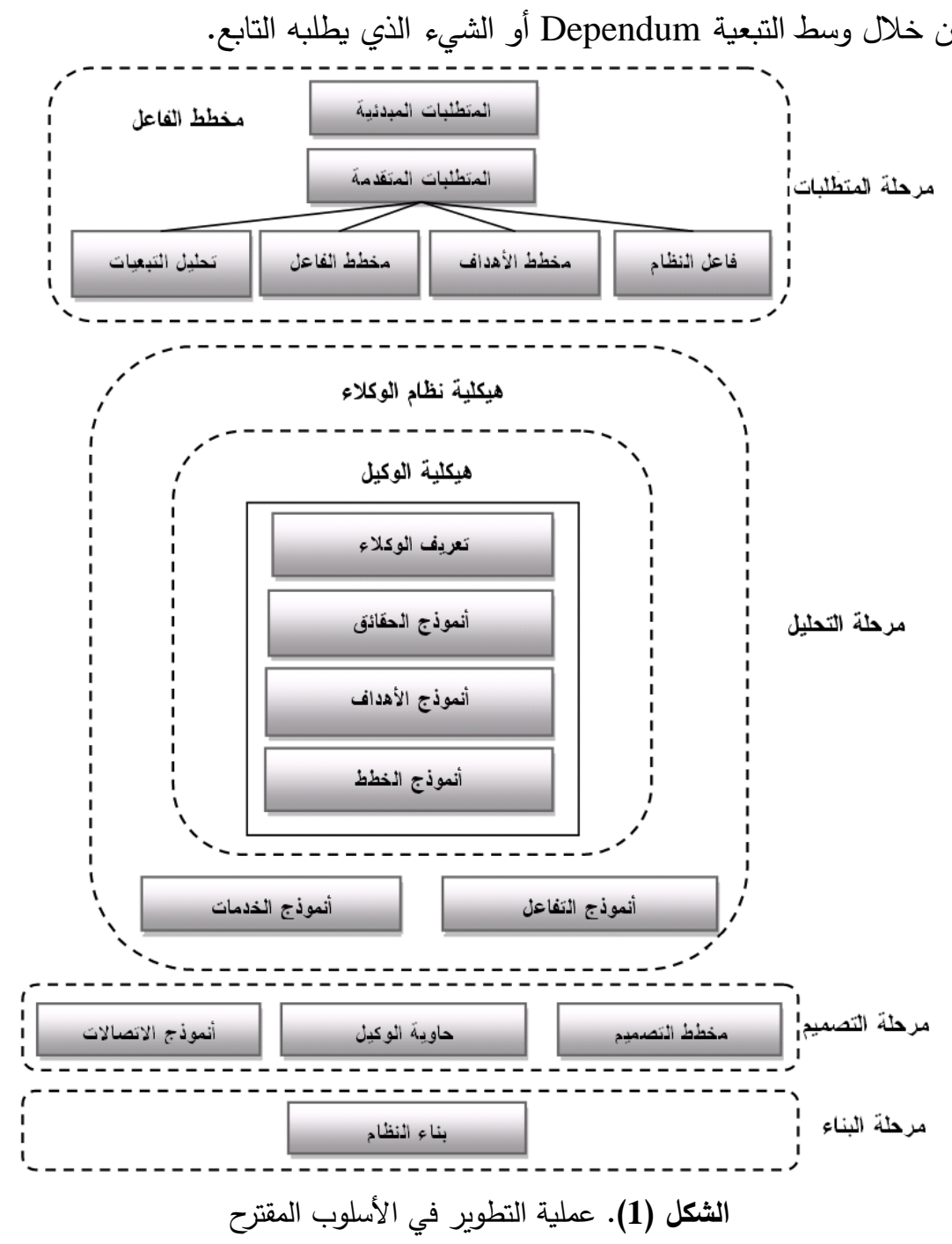

تحتوي مرحلة المتطلبات على الترميزات الخاصة بجميع المكونات والتي تستخدم في مخطط الفاعل.

يوضح الشكل (2) الترميزات المستخدمة في مخطط الفاعل.

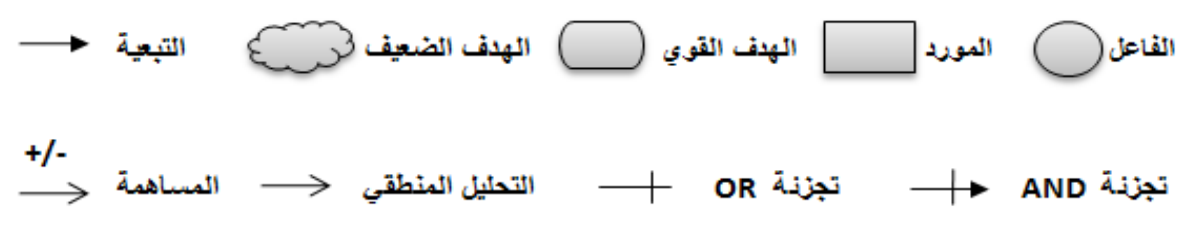

الثكل (2). الترميزات المستخدمة في مخطط الفاعل

تحتوي مرحلة المتطلبات على مرحلتين وهما مرحلة المتطلبات المبئية والمتقدمة:

1- المتطلبات المبئية Early Requirements

في مرحلة المتطلبات المبدئية يتم فهم لنطاق النظام بصورة عامة وتمثيله بمخطط بسيط وهو مخطط الفاعل المبئي، ويتميز هذا المخطط بالبساطة والوضوح للمستخدم. تم وضع المخطط البسيط في هذه المرحلة 
وذلك لمشاركة الزبون أو المستخدم النهائي في هذه المرحلة، ولكي تكون المتطلبات واضحة للزبون والمطور وفهم احتياجات الزبون بصورة صحيحة. الثكل (3) في جهة اليمين يوضح مخطط الفاعل المبدئي لتطبيق التجارة الالكترونية.
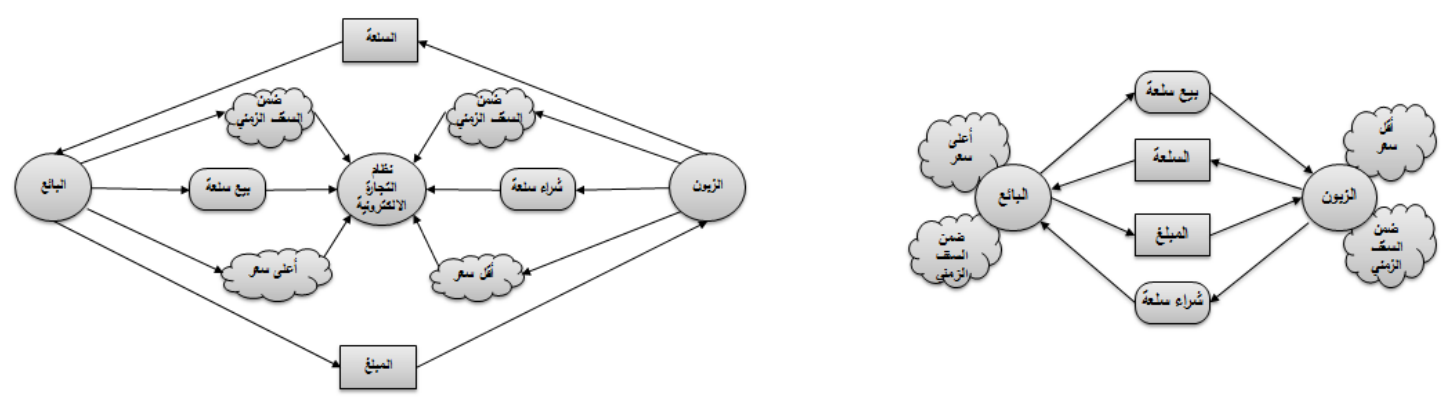

الثكل (3). مخطط الفاعل المبدئي لتطبيق التجارة الالكترونية

Late Requirements المتطلبات المتقدمة

تتكون مرحلة المتطلبات المتقدمة من أربع خطوات وهي إدخال فاعل النظام إلى الأنموذج وتكوين مخطط الأهداف وتكوين مخطط الفاعل النهائي وتحليل التبعيات.

أ- فاعل النظام System Actor

الخطوة الأولى لمرحلة المتطلبات المتقدمة هي إدخال الفاعل الذي يمثل النظام قيد التطوير ، ويتم تكرار

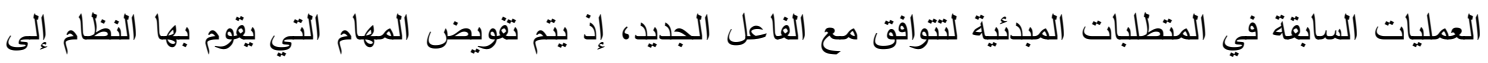

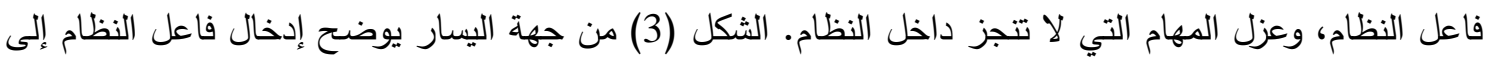
مخطط الفاعل المبئي لتطبيق التجارة الالكترونية.

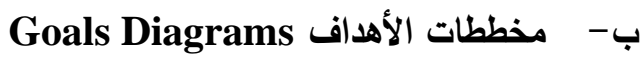

في الخطوة الثانية يتم تكوين مخططات الأهداف وتتمحور هذه الخطوة بثلاث مراحل وهي، تجزئة الأهداف Goal Decomposition، يتم في هذه المرحلة تجزئة الهدف أو الأهداف إلى أهداف فرعية بطريقة تجزئة AND أو تجزئة OR لتكوين شجرة من الأهداف ولكل فاعل على حدة. التحليل المنطقي للأهداف لتهني Means-end Analysis الهدف مروراً بجميع الأهداف الفرعية والأهداف الضعيفة والمهام والموارد اللازمة لتحقيق هذا الهدف. تحليل مساهمة الأهداف Contribution Analysis يتم في هذه المرحلة تحليل مساهمة الأهداف التي تساهم بشكل ايجابي أو سلبي في تحقيق أهداف أخرى.

\section{ج- مخطط الفاعل النهائي هiagram}

يتم في هذه الخطوة تجميع المخططات الخاصة بفاعل النظام والأهداف لتكوين مخطط الفاعل النهائي لمرحلة المتطلبات والذي يحتوي على جميع مكونات النظام، ويتم رسم حدود لتمييز المهام الخاصة بأهداف الفاعلين التي يؤديها النظام. يوضح الثكل (4) مخطط الفاعل النهائي لتطبيق التجارة الالكترونية. 


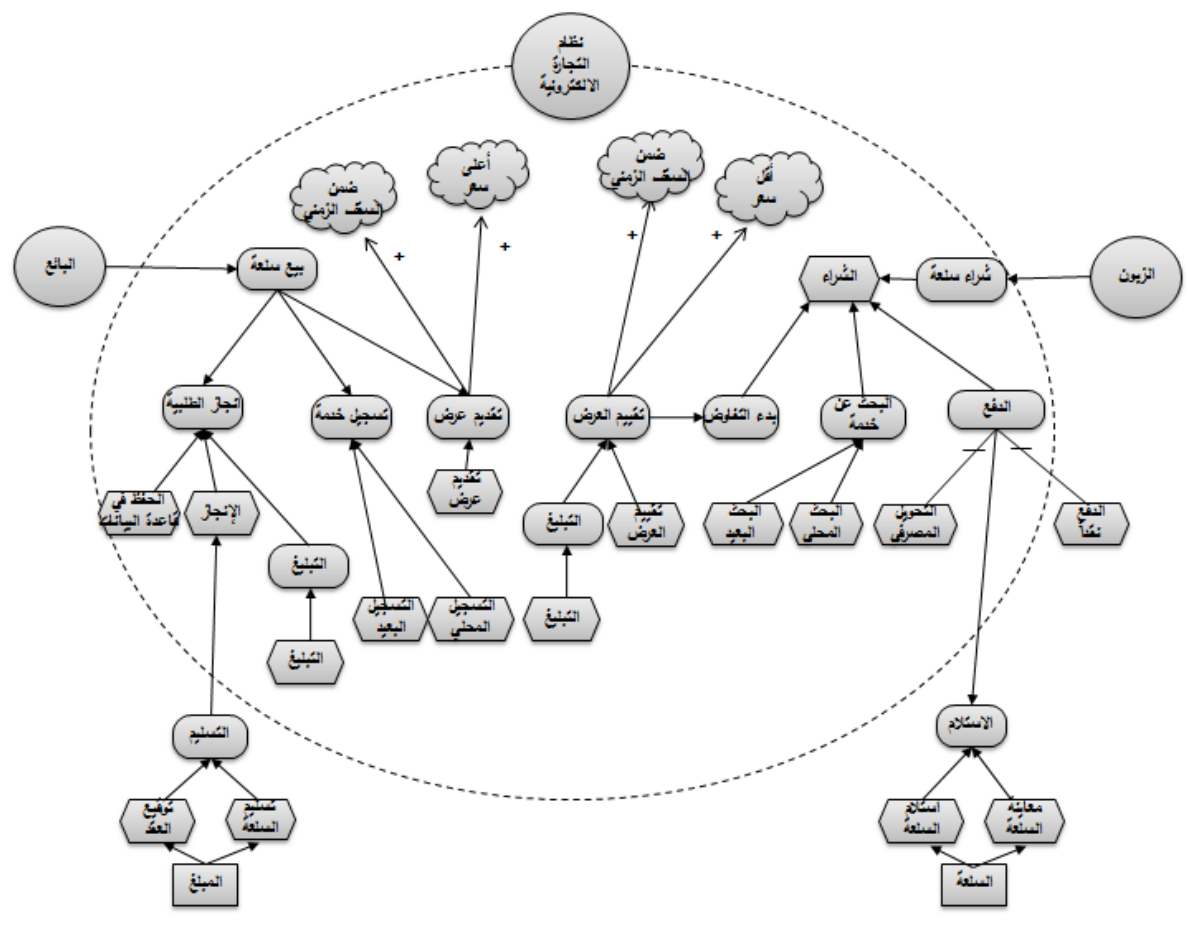

الشكل (4). مخطط الفاعل لتطبيق التجارة الاكترونية

Dependency Analysis دـ - تحليل التبعيات

يتم في هذه الخطوة تحليل التبعيات بين الفاعلين من خلال الأهداف والمهام والموارد لتحديد الأولويات والثروط السابقة واللاحقة لتسلسل تتفيذ الأهداف والمهام ولتكوين المشغلات التي تقرر أي من الأهداف ومتى سوف يبدأ.

2-8 مرحلة التحليل Analysis Phase

الهذف من مرحلة التحليل هو تحويل متطلبات النظام بشكل واضح لتمثيل النظام بشكل مبدئي وتهيئته

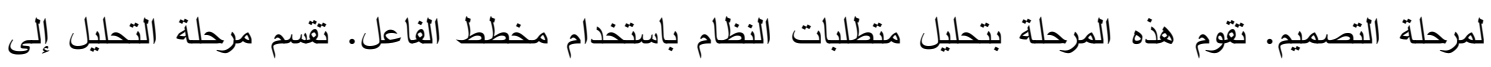
خطوتين رئيسيتين، الأولى هي تكوين وصف لهيكلية الوكيل بثكل منفرد، والثانية هي وصف للنظام بشكل كلي.

\section{1-1 هيكلية الوكيل Agent Architecture}

يتم في هذه الخطوة تعريف أنموذج الوكيل الذي يصف الهيكلية الداخلية للوكلاء داخل النظام وكيفية قدرة هؤلاء

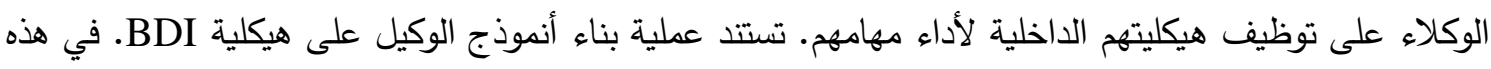

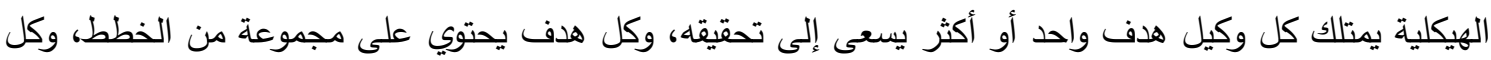
خطة تتضمن مجموعة من المهام. يمتلك الوكلاء أيضاً المشغلات التي تساعدهم على تحديد الهدف أو الخطة المناسبين ليتم اختياره. تحتوي هذه الخطوة على أربع خطوات فرعية وهي تعريف الوكلاء، تكوين نماذج الحقائق، تكوين نماذج الأهداف، وتكوين نماذج الخطط.

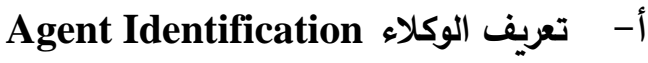

يتم في هذه الخطوة تعريف الوكلاء في النظام وتعيين الأدوار اللازمة لهم. يتم الكثف عن الوكلاء من خلال

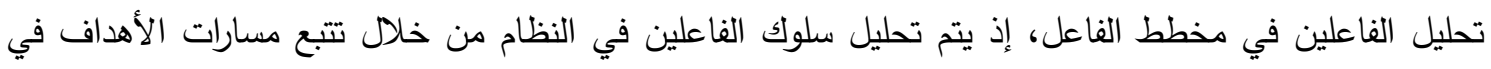

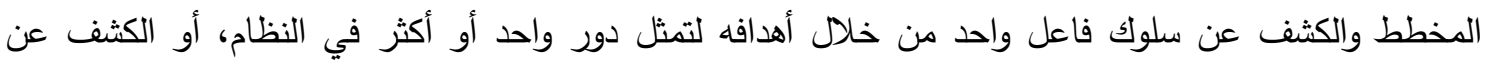


سلوك أكثر من فاعل يقومون بنفس الدور من خلال أهدافهم لتمثل دور واحد في النظام. حيث يمكن بهذه العملية أن يكون كل فاعل على شكل وكيل واحد، أو من خلال دمج عدة فاعلين لتكوين وكيل واحد، وفي هذه الحالة يتم

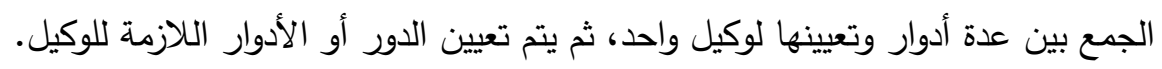

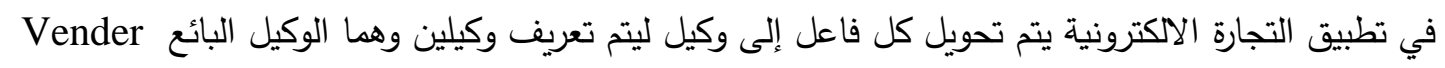
Agent والوكيل المشتري Customer Agent، بالإضافة إلى تعيين الأدوار المستخرجة لكل منهما. يوضح الشكل (5) تعيين الأدوار للوكلاء.

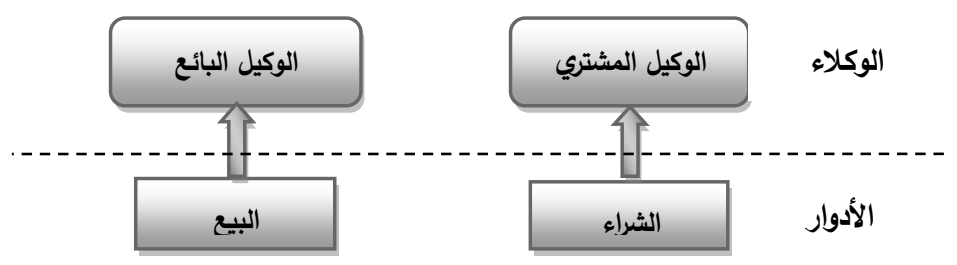

الثكل (5). تعيين الأدوار للوكلاء في تطبيق التجارة الاككترونية

بeliefs Model ب - بموذج الحقائق حقائق الوكيل تمثل معلومات الوكيل عن بيئته أو عن نفسه أو غيره من الوكلاء [7]. يتم تعريف حقائق

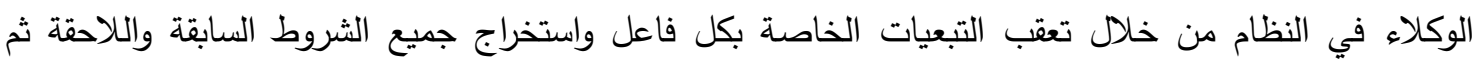

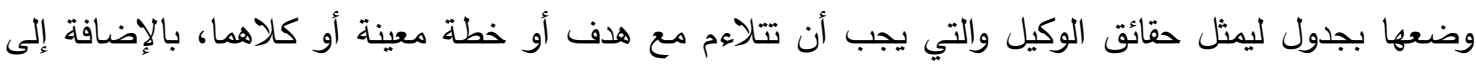
ذلك، الحقائق تخزن معلومات حول الحالة الداخلية للوكلاء. تصنف الحقائق على النحو التالي، الحقيقة المخزونة Storage Belief دورة حياته. الحقيقة المحافظة Maintain Belief، يستخدم هذا النوع عندما يحافظ الوكيل على قيمة الحقيقة. حقيقة الإنجاز Achieve Belief، يستخدم هذا النوع عندما يخزن الوكيل قيمة مطلوبة من الحقيقة، وخلال دورة

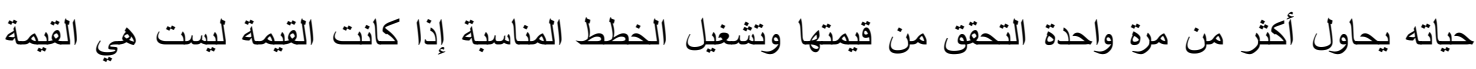

المطلوبة [6]. ويتم تصنيف الحقائق حسب نوع القيمة إذا كانت ثابتة أو متغيرة.

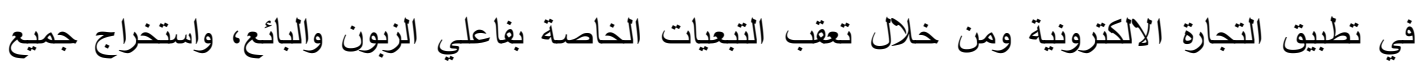
الشروط السابقة واللاحقة، يتم إعداد أنموذج حقائق الوكيل المشتري وأنموذج حقائق الوكيل البائع. يوضح التحن الجدول (1) أنموذج حقائق الوكيل المشتري.

\begin{tabular}{|c|c|c|}
\hline الغرض الغ & النوع & أسم الحقيقة \\
\hline للحفظ & ثابت & أسم الزبون \\
\hline للحفظ & متغير & أسم البائع \\
\hline للحفظ & 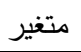 & تم إدخال السلع الدطلوب شراؤها \\
\hline للحفظ & 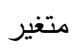 & السلعة معروضة للبيع \\
\hline 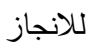 & متغير & تم العثور على خدمة \\
\hline للحفظ & متغير & تم إرسال الطلب \\
\hline للحفظ & متغير & تم إرسال العرض \\
\hline للحفظ & 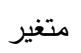 & قبول العرض \\
\hline 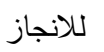 & 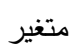 & إنجاز الطلبية \\
\hline
\end{tabular}




\begin{tabular}{|c|c|c|}
\hline للحفظ & متغير & تم دفع المبلغ \\
\hline للحفظ & متغير & تم تبليغ المستخدم الحقيقي \\
\hline للحفظ & ثابت & الخدمة \\
\hline للحفظ & متغير & السلعة \\
\hline للحفظ & متغير & تفاصيل المفاوضات \\
\hline للحفظ & متغير & الوقت \\
\hline للحفظ & متغير & واجهة الوكيل GUI \\
\hline
\end{tabular}

ج- أنموذج الأهداف Goals Model يمثل الهذف حالة مستهدفة يحاول الوكيل تحقيقها. والهذف يكون سبب تنفيذ إجراءات الوكيل. هناك

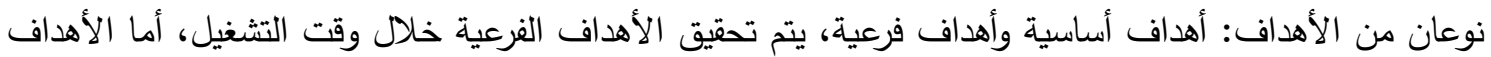
الأساسية فهي الأهداف الإستراتيجية للنظام [6]. يتم تعريف الأهداف في الأنموذج ولكل وكيل في النظام، ويتم ذلك من خلال تحويل الأهداف الخاصة بالفاعل في أنموذج الفاعل ضمن دوره في هذا الأنموذج وتتقل إلى أنموذج أهدافه، وأيضاً تحويل مهام هذا الدور إلى الخطط الخاصة بهذا الهدف، بالإضافة إلى تحويل الثروط السابقة واللاحقة التي تم تعريفها في مرحلة تحليل التبعيات الخاصة بالهدف إلى أنموذجه.

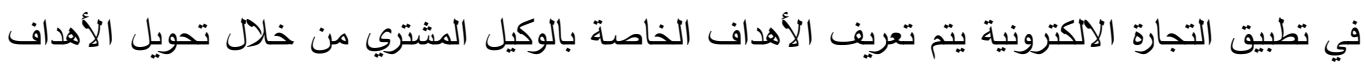

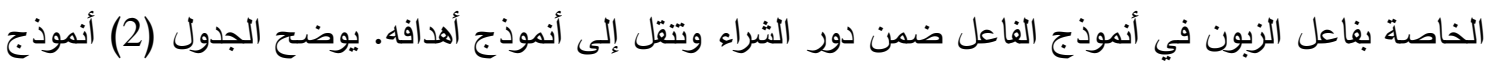
الأهداف للوكيل المشتري.

الجدول (2). أنموذج الأهداف للوكيل المشتري

\begin{tabular}{|c|c|c|c|}
\hline الخطط & الثرط اللاحق & الشرط السابق & أسم الهدف \\
\hline • الشراء & • إنجاز الطلبية & • • السلعة معروضة للبيع & شراء سلعة \\
\hline • البحث عن الخدمة & • • لم العثور على خدمة العثور على خدمة & • تم إدخال السلع المطلوب شراؤها & البحث عن خدمة \\
\hline • تقييم العرض & • تم إرسال الطلب & • تم العثور على خدمة & بدء التفاوض \\
\hline • • • • • & • • قبول العرض العرض & • تم إرسال العرض & تقييم العرض \\
\hline • التحويل المصرفي & • • • • لم دفع المبلغ دفع المبلغ & • • إنجاز الطلبية & 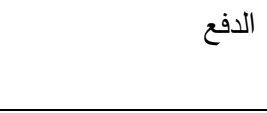 \\
\hline • • • • • تبليخ بالاتفاق والسعر بموعد الاستلام & • تم تبليخ المستخدم الحقيقي & • • إنجاز الطلبية & تبليغ المستخدم الحقيقي \\
\hline
\end{tabular}

Plan Model د أنموذج الخطة

لتتفيذ أهداف الوكيل هناك خطة أو مجموعة من الخطط لكل هدف لغرض تنفيذها من خلال آلية معينة، فالخطة هي وسيلة يمكن من خلالها تحقيق أهداف الوكلاء، يتم تعريف الخطط من خلال تحويل المهام التابعة للأهداف في مخطط الفاعل، أو مباشرة من أنموذج الأهداف. 
في تطبيق التجارة الالكترونية ومن خلال تتبع أنموذج أهداف الوكيل المشتري، يتم تكوين نماذج الخطط الخاصة بهذا الوكيل، وبعدها يتم تنقية هذه الخطط من خلال دمج الخطط ذات المهام المتثابهة، أو تجزئة الخطة

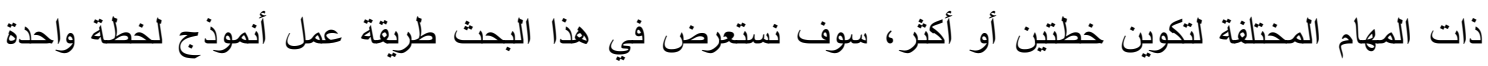
فقط. يوضح الجدول (3) خطة الثراء الخاصة بالوكيل المشتري.

الجدول (3). خطة الشراء للوكيل المشتري

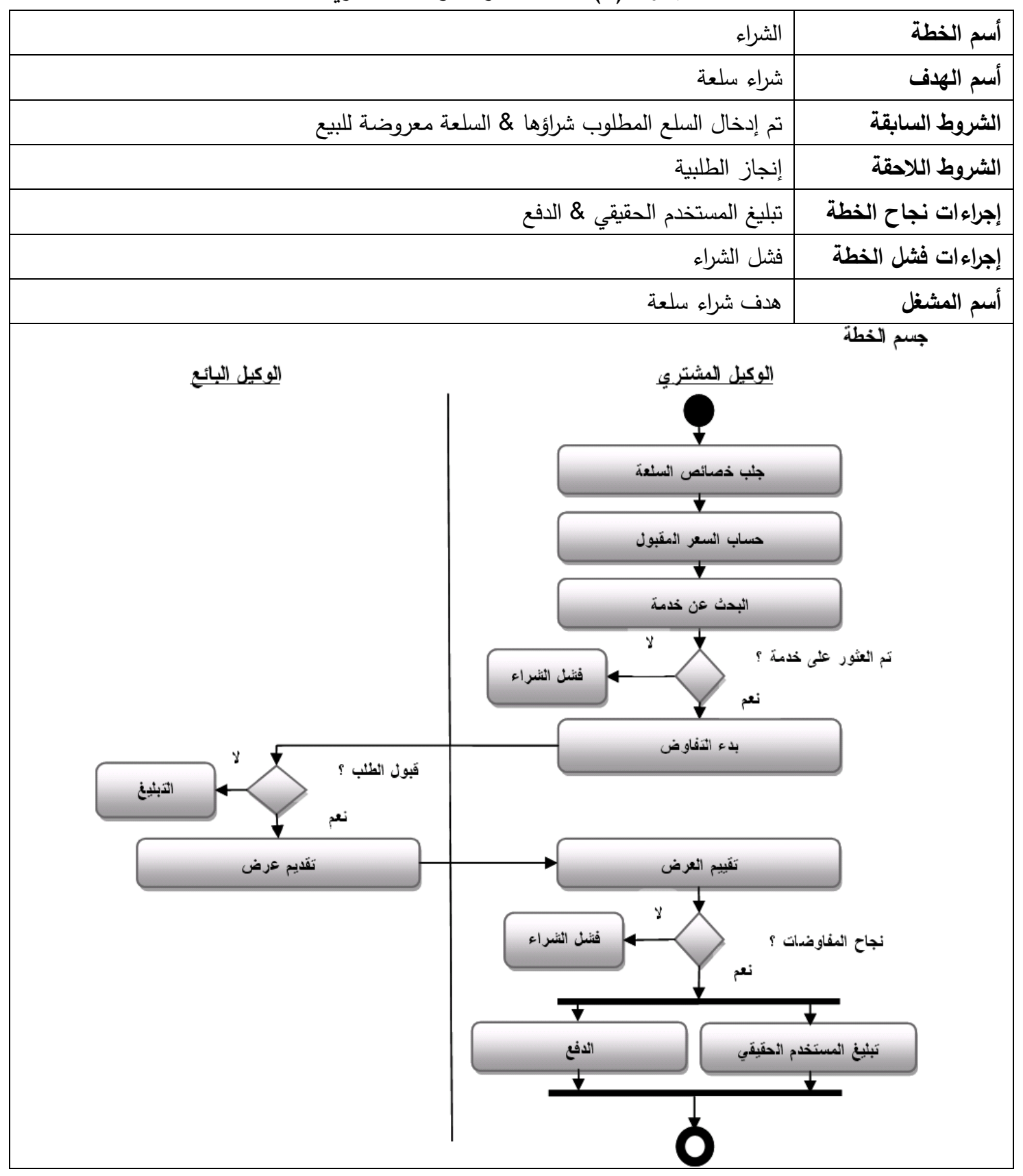


2-كلية نظام الوكلاء MAS Architecture

وهي الجزء الثاني من مرحلة التحليل ويتم فيها تصميم مخطط التفاعل الذي يتم من خلاله الكثف عن جميع التفاعلات بين الوكلاء في النظام. يتم بعد ذلك تصميم أنموذج الخدمات، الذي يتم من خلاله تعريف جميع

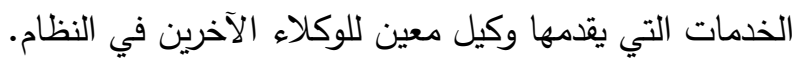

أ- مخطط التفاعل Interaction Diagram وهو مخطط يصور التفاعل بين جميع الوكلاء في النظام، ويوضح عملية تبادل المعلومات بين الوكلاء مع بعضهم البعض ومع بيئتهم، وهو يصف المحادثات بين الوكلاء على شكل عالي المستوى من التفاعلات ويعد كخطوة أولية لتصميم المراسلات بين الوكلاء. يتم تصميم مخطط التفاعل من خلال الكثف عن التفاعلات الموجودة بين

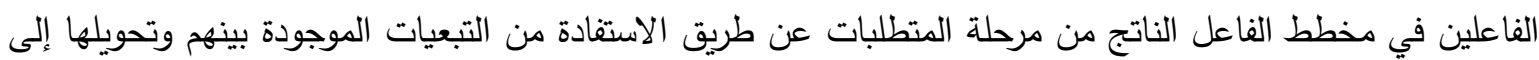
محادثات. يوضح الثكل (6) أنموذج التفاعل بين الوكيل المشتري والوكيل البائع في تطبيق التجارة الالكترونية.

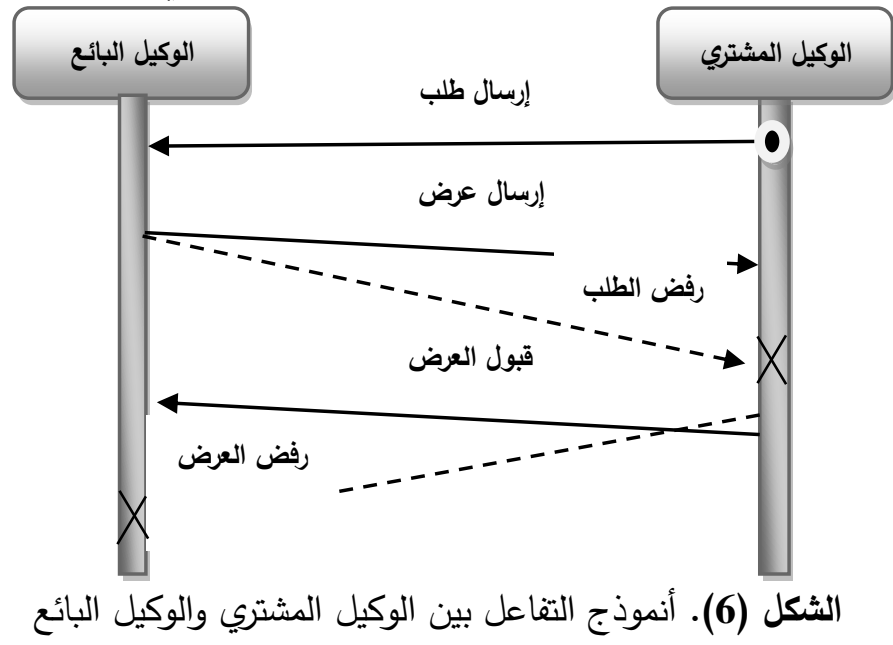

Directory Facilitator Model بـ - أنموذج الخدمات

يتم في هذه المرحلة إعداد أنموذج دليل الخدمات في النظام، إذ يستخدم دليل الخدمات لعرض الخدمات التي يوفرها بعض الوكلاء في النظام مع المعلومات والوصف الخاص بها والوكيل الموفر للخدمة لكي يستطيع الوكلاء الآخرون تمييز الخدمات التي يعرضها النظام. يتم الكثف عن الخدمات من خلال مخطط الفاعل، إذ يعد الفاعل الذي يكون متبوعاً هو الوكيل موفر الخدمة، والوكلاء التابعين له في تحقيق الهدف الأساسي لهم يعدون الوكلاء المستفيدين من الخدمة. في تطبيق التجارة الاككترونية تم وضع خدمة (المبيعات) التي يسجلها الوكيل البائع لكي يستطيع الوكلاء المشترون من العثور على الخدمة وتقديم طلب الشراء، بالإضافة إلى ذلك وجود خدمة اختيارية ثانية وهي (المساعدة) التي تستخدم لوصف طريقة البيع والشراء في هذا التطبيق. يوضح الجدول (4) أنموذج خدمات تطبيق التجارة الاككترونية. 


\begin{tabular}{|c|c|c|c|c|c|}
\hline \multicolumn{6}{|c|}{ الجدول (4). أنموذج خدمات تطبيق التجارة الاككترونية } \\
\hline 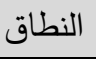 & 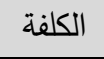 & 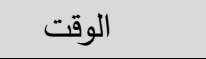 & تاريخ انتهاء الخدمة & 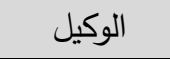 & 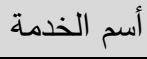 \\
\hline بعيد & 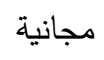 & دائماً & دائماً & 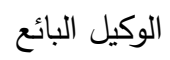 & 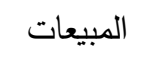 \\
\hline بعيد & 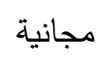 & 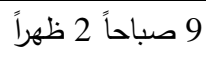 & دائماً & الوكيل البائع & المساعدة \\
\hline
\end{tabular}

\section{Design Phase 3-8 مرحلة التصميم}

يتم في هذه المرحلة تمثيل النماذج التي تم بناؤها في مرحلة التحليل بصورة مفصلة، إذ يتم مراجعة هذه النماذج

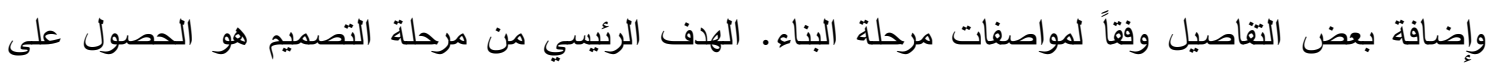

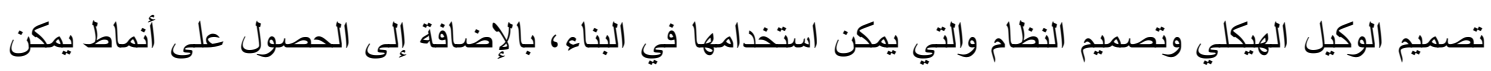

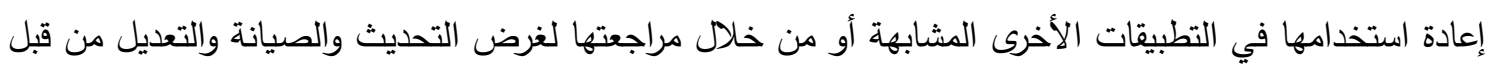
مهندس البرمجيات، والكثير من فوائد التصميم الأخرى. تتمحور هذه المرحلة في ثلاث خطوات: إنثاء مخطط التصميم، تعريف حاوية الوكيل، تعريف أنموذج الاتصالات.

\section{1- مخطط التصميم Design Diagram}

يتم في الخطوة الأولى تعريف الهيكلية الثاملة للنظام من خلال تجزئته إلى نظم فرعية وتعريف العلاقات بين هذه النظم التي تكون معتمدة على الموارد والمهام، وهذه النظم الفرعية تكون مترابطة من خلال البيانات

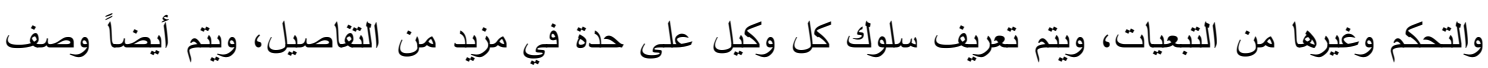

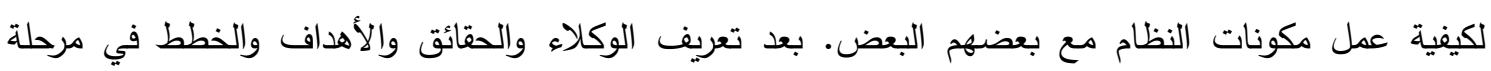
التحليل، يمكن إعادة تثكيل مخطط الفاعل الذي تم إعداده في مرحلة المتطلبات لتكوين مخطط التصديم التصفيم الذي يتم من خلاله وصف الوكلاء في النظام وأهداف وخطط كل واحد منهم بالإضافة إلى عملية التواصل بينهم. الثكل

$$
\text { (7) يوضح مخطط تصميم تطبيق التجارة الالكترونية. }
$$

\section{Agent Container 2- 2 حاوية الوكيل}

الخطوة الثانية من مرحلة التصميم هي بناء حاوية الوكيل وهي التي تمثل سلوك الوكيل في النظام. يوجد هدفين أساسيين لحاوية الوكيل، الأول هو تجميع الحقائق والأهداف والخطط الخاصة بوكيل وهيل واحئ واحد ليكون نظاماً فرعياً في النظام الأساسي، والهدف الثاني هو تجميع الحقائق والأهداف والخطط المتكررة في أكثر من وكيل ووضعها في حزمة واحدة تسمى المقدرة Capability وهي التي يمكن أن يستدعيها جميع الوكلاء بدلاً من تكرار

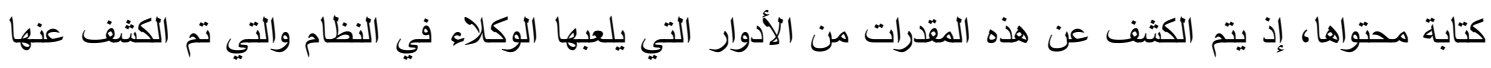

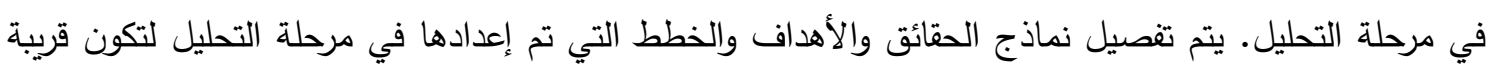
لعملية البناء ولتتوافق مع الإطار أو المنصة التي يتم اختيارها لغرض البناء، وفي هذا البحث سيتم إنشاء النماذج

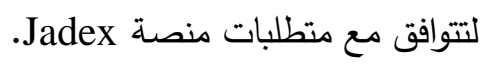

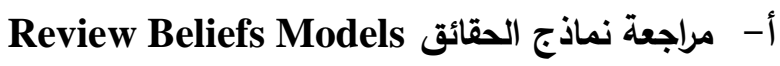

يتم مراجعة نماذج الحقائق في مرحلة التحليل وتحديثها لتتلاءم مع مواصفات التصميم بإضافة حقل الفئة الذي

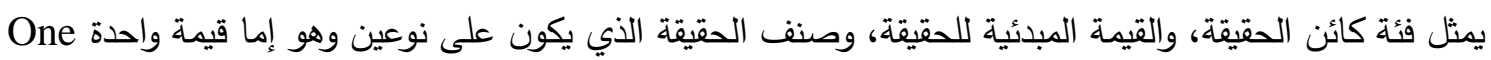


أو مجموعة قيم Set، والاسم التعريفي Identifier الذي يستخدم في مرحلة البناء. الجدول (5) يوضح الإضافات لأنموذج حقائق الوكيل المشتري.

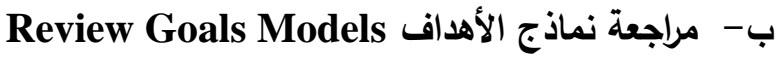

يتم في هذه المرحلة مراجعة أنموذج الأهداف الذي تم إعداده في مرحلة التحليل وتحديثه ليتلاءم مع مواصفات التصميم، إذ يتم إضافة حقلي الاسم التعريفي الذي يستخدم في مرحلة البناء، وحقل النوع لتصنيف أهداف الوكيل

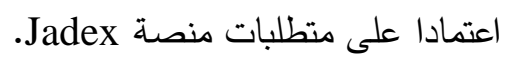
أن الأهداف في منصة Jadex تصنف إلى أربعة أنواع وهي، هدف الأداء Perform Goal، هدف الانجاز

Achieve Goal

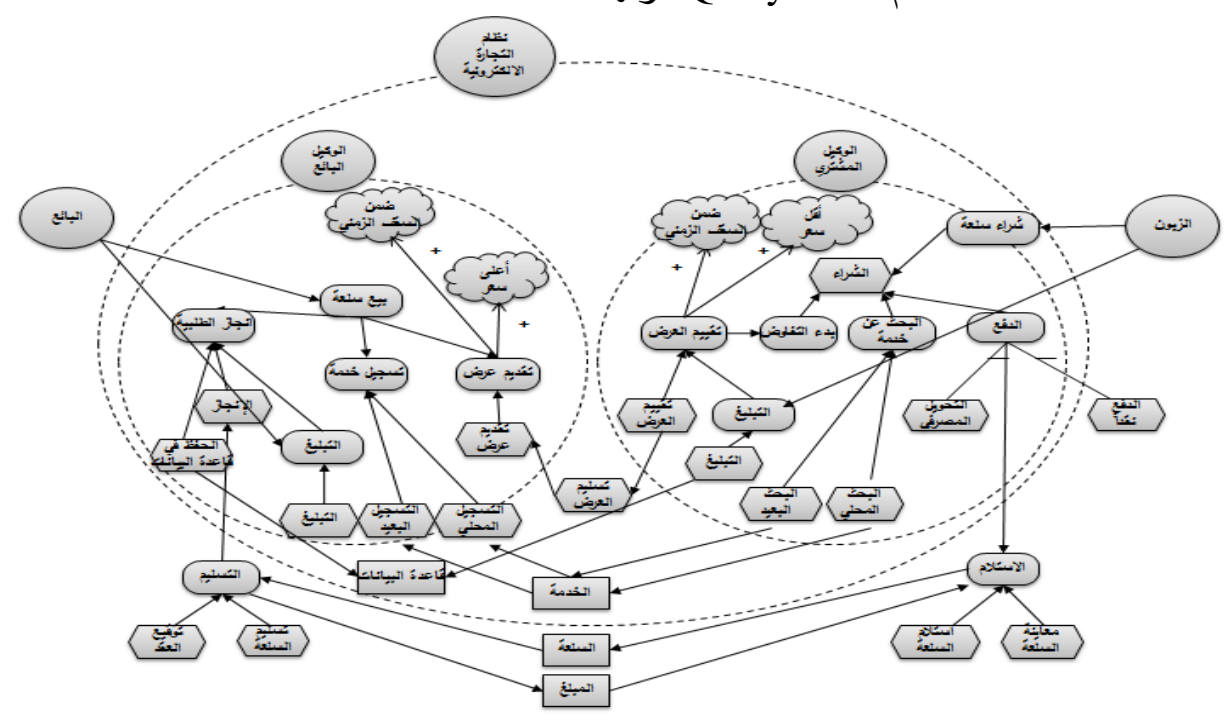

الثكل (7). مخطط تصميم تطبيق التجارة الاككترونية

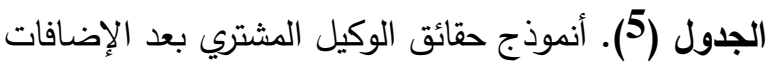

\begin{tabular}{|c|c|c|c|c|c|c|}
\hline الصنف & القيمة المبائية & الفئة & الاسم التعريفي & الغرض & النوع & أسم الحقيقة \\
\hline One & Customer & String & customerName & للحفظ & ثابت & أسم الزبون \\
\hline Set & Vender & String & vanderName & للحفظ & 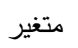 & أسم البائع \\
\hline One & False & Boolean & orderAdded & للحفظ & متغير & تم إدخال السلع المطلوب شراؤها \\
\hline One & False & Boolean & openState & للحفظ & 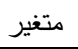 & السلعة معروضة للبيع \\
\hline One & False & Boolean & dfFound & ل للانجاز & متغير & تم العثور على خدمة \\
\hline One & False & Boolean & requestSent & للحفظ & متغير & تم إرسال الطلب \\
\hline One & False & Boolean & offerSent & للحفظ & متغير & تم إرسال العرض \\
\hline One & False & Boolean & acceptOffer & للحفظ & متغير & قبول العرض \\
\hline One & False & Boolean & doneState & ل للانجاز & متغير & إنجاز الطلبية \\
\hline One & False & Boolean & moneyPaid & للحفظ & متغير & تم دفع المبلغ \\
\hline One & False & Boolean & rcNotified & للحفظ & متغير & تم تبليغ المستخدم الحقيقي \\
\hline Set & Null & Order & Orders & للحفظ & ثابت & السلع ـ \\
\hline One & مبيعات & String & dfServiceName & للحفظ & متغير & الخدمة \\
\hline Set & Null & Report & Reports & للحفظ & متغير & تفاصيل المفاوضات \\
\hline One & CurrTime & Long & Time & للحفظ & متغير & الوقت \\
\hline One & Constructure & Gui & Gui & للحفظ & متغير & GUI واجهة الوكيل \\
\hline
\end{tabular}


هدف الأداء هو نوع من الأهداف الذي يتطلب بعض الإجراءات لتتفيذه، ونتيجة الهدف تعتمد على إجراءات

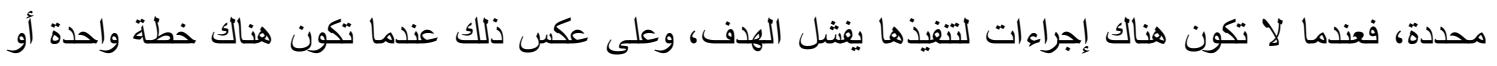
أكثر تتنفذ ينجح الهدف. هدف الانجاز هو نوع من الأهداف الذي يستخدم عندما يريد الوكيل تحقيق حالة مستهدفة

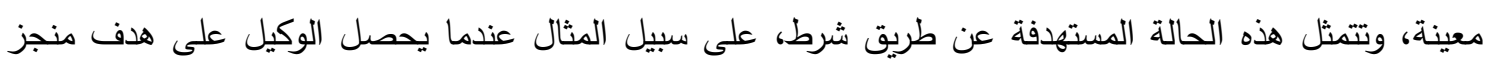

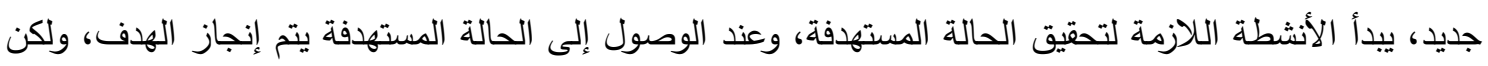
إذا لم ينجز إلى حد الآن يتم اختيار الخطط اللازمة لتتفيذها، وهكذا إلى حين الوصول إلى إلى الحالة المستهدفة يتم

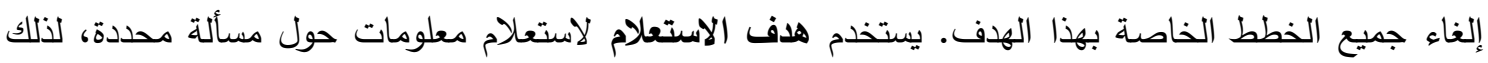
يستخدم هذا الهدف لاسترداد نتيجة الاستعلام. الهدف المحافظ هو الهدف الذي يحافظ على حالة معينة، أي لأي

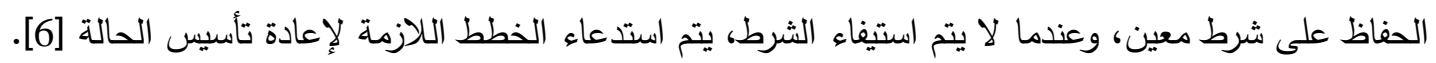
في تطبيق التجارة الاكترونية يتم إضافة حقلي الاسم التعريفي والنوع لتصنيف أهداف الوكيل في أنموذج الأهداف. الجدول (6) يوضح أنموذج أهداف الوكيل المشتري بعد إضافة الحقول الجديدة.

\begin{tabular}{|c|c|c|c|c|c|}
\hline 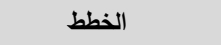 & الشرط اللاحق & الثرط السابق & الاسم التعريفي & النوع - اع & أسم الهذف \\
\hline • • | لمراء & • إنجاز الطلبية & • السلعة معروضة للبيع & buyGoal & 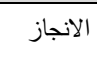 & شراء سلعة \\
\hline • • البحث عن الخذمة & • • لم يتم العثور على خلى خدمة & • • إذال السلع المطلوب شراؤها & dfSearch & الانجاز & البحث عن خما \\
\hline • تقييم العرض & • • تم إرسال الطلب & • تم العثور على خدمة & cnpInitiate & الانجاز & بدءء التفاوض \\
\hline • • ت تقييم العروض العرض & • • قبوض العرض & • • تم إرسال العرض & $\begin{array}{l}\text { cnpEvaluate } \\
\text { proposals }\end{array}$ & الاستعلام & تقييم العرض \\
\hline • • التحويل المصرفي & • • • تم دفتم دفع المبلغ & • إنجاز الطلبية & payGoal & الانجاز & الدفع \\
\hline 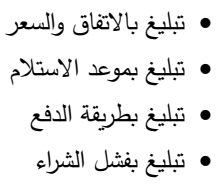 & • • تم تبليخ المتخذم الحقيقي & • • إنجاز الطلبية & notifyGoal & الانجاز & تلبيغ المستخدم \\
\hline
\end{tabular}

ج- مراجعة نماذج الخطط Review Plans Models

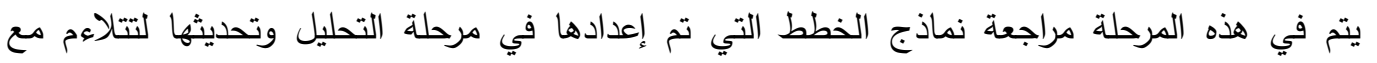

مواصفات التصميم، إذ يتم إضافة حقلي الاسم التعريفي الذي يستخدم في مرحلة البناء، وحقل النوع لتصنيف خطط الوكيل اعتماداً على متطلبات منصة Jadex. ذلك أن الخطط تصنف إلى نوعين: خطط الخدمة Service Plans والخطط السلبية Passive Plans. خطة الخدمة لديها طبيعة خدمية، نسخة Instance هذه الخطة تتتفذ وتتنظر

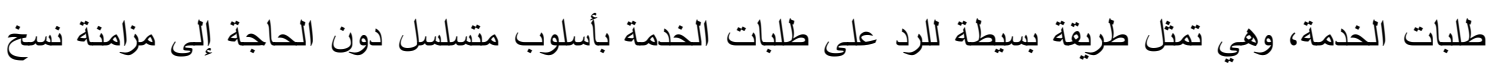

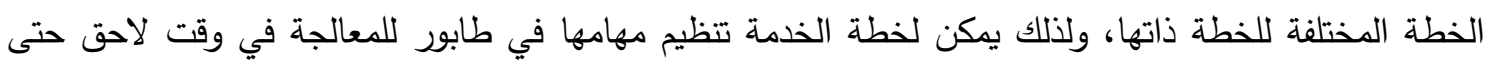
عندما تكون مشغولة. يتم تثغيل الخطة السلبية فقط عندما يكون لديها مهمة لتحقيقها، وفي هذا النوع من الخطط يجب أن يتم تحديد الأهداف وأحداث المشغلات للسماح للوكيل بمعرفة أنواع الأحداث التي يمكن للخطة أن تتعامل 
معها. عندما يستلم الوكيل حدث معين، يجب تحديد خطة أو خطط مرشحة وإنشاء نسخ لها لتنفيذها [6]. الجدول (7) يوضح أنموذج خطة الشراء للوكيل المشتري بعد إضافة الحقول الجديدة. الجدول (7). أنموذج خطة الثراء للوكيل المشتري بعد إضافة الحقول الجديدة

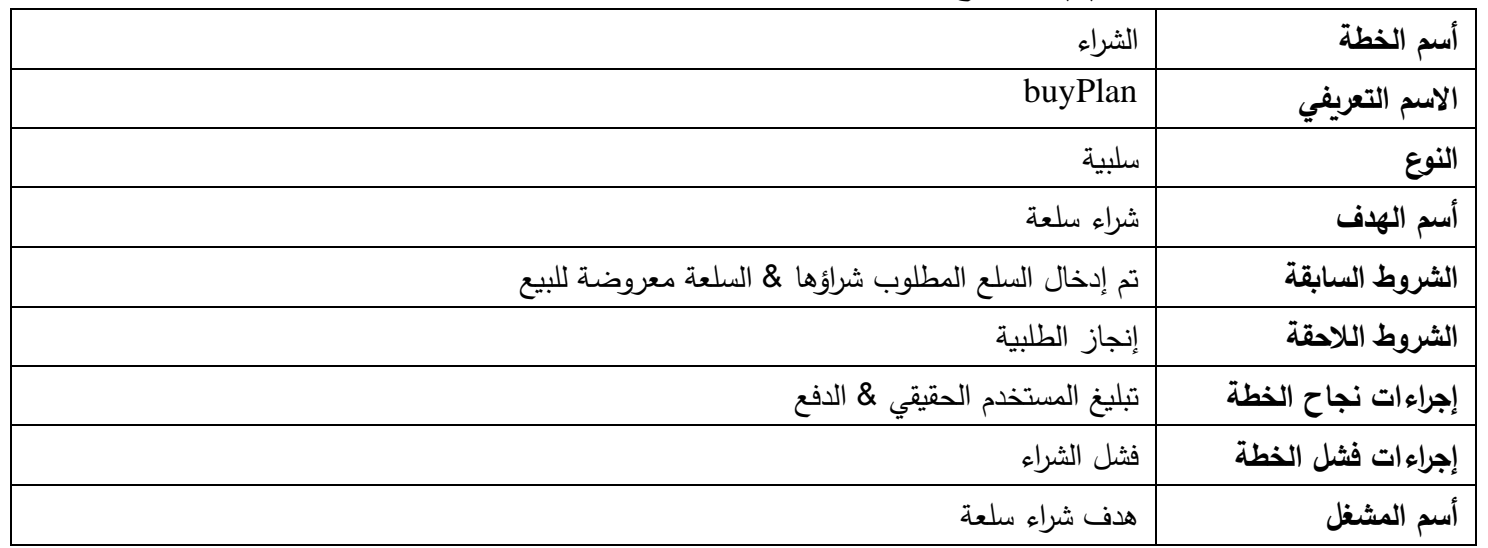

\section{Communication Model 3- أنموذج الاتصالات}

في الخطوة الثالثة من مرحلة التصميم يتم إعداد أنموذج الاتصالات، وهذا الأنموذج يصف بالتفصيل

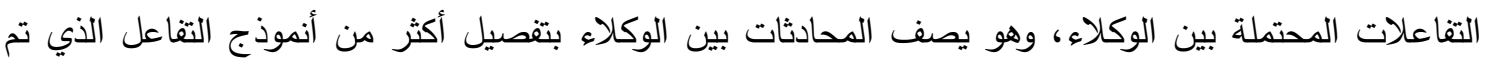
إنثاؤه في مرحلة التحليل. يتم إعداد أنموذج الاتصالات من خلال تحويل أنموذج التفاعل الذي تم إعداده في مرحلة التحليل إلى رسائل المحادثة وفقا لأحد بروتوكولات FIPA. ويتم إعداد صيغة الرسائل المتبادلة بين الوكلاء في

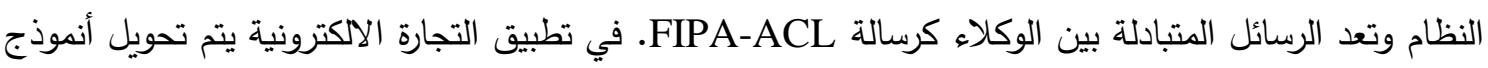
التفاعل إلى بروتوكول التفاوض FIPA Contract Net Protocol [17]، وذلك للاستفادة من خصائص هذا البروتوكول. الثكل (8) يوضح تحويل أنموذج التفاعل إلى بروتوكول التفاوض.

\section{Implementation Phase مرحلة البناء 4 -8}

مرحلة البناء هي عملية التطوير الفعلية من خلال بدء كتابة الرموز البرمجية للبرنامج. يتم في هذه المرحلة

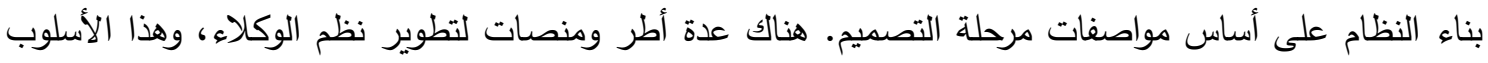

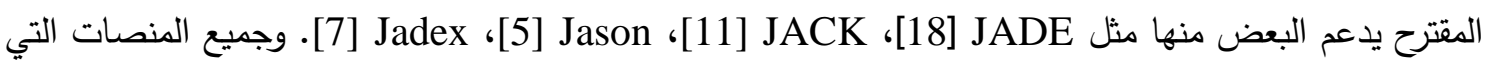

تستخدم هيكلية BDI ومقاييس FIPA. في هذا البحث سوف نستعرض مرحلة البناء باستخدام منصة Jadex. أ- بناء حاوية الوكيل الخطوة الأولى من عملية البناء هي تحويل حاوية الوكيل التي تم إعدادها في مرحلة التصميم إلى ملف يسمى ملف تعريف الوكيل Agent Definition File (ADF) وهو عبارة عن ملف مكتوب بلغة XML 


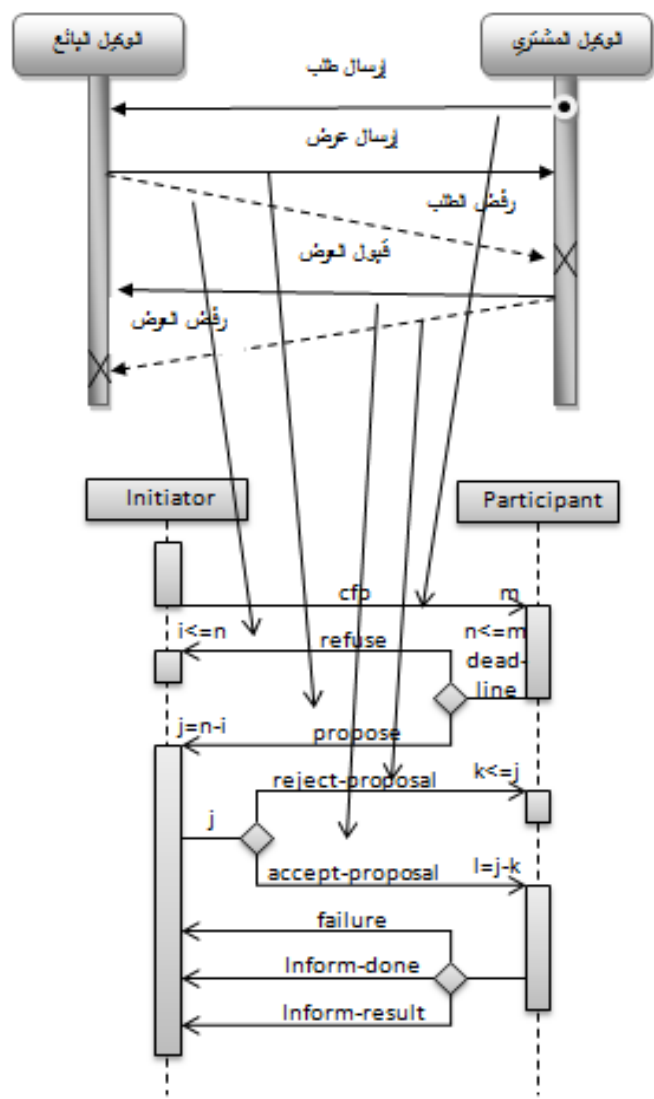

FIPA Contract Net الثكل (8). تحويل أنموذج التفاعل إلى بروتوكول

يحتوي على جميع خصائص الوكيل، ويتم تكوين هذا الملف لكل وكيل في النظام. تخضع لغة XML في هذا الملف إلى محددات Jadex Schema المحددة مسبقاً من المنصة. بعد تكوين ملف ADF يتم تحويل الحقائق الموجودة في أنموذج الحقائق الخاصة بالوكيل إلى رموز برمجية بلغة XML في هذا الملف، وبالطريقة نفسها يتم تحويل الأهداف الموجودة في أنموذج الأهداف وتحويل جزء رأس الخطط من نماذج الخطط.

ب- - بناء جسم الخطط

بعد عملية بناء ملف ADF، يتم في الخطوة الثانية بناء فئات لغة الجافا من خلال تحويل مخططات الفعالية الموجودة في جزء جسم الخطة في نماذج الخطط إلى الرموز البرمجية بلغة جافا، كل فئة تحفظ في ملف مستقل وبنفس أسم الفئة.

\section{7- تنفيذ تطبيق التجارة الاكترونية}

بعد تشغيل منصة Jadex في جهاز الخادم يتم تتفيذ التطبيق وظهور واجهة المستخدم الخاصة بالبائع، يقوم البائع بتسمية الخذمة المراد تسجيلها وإدخال السلع التي يرغب في بيعها، وفي الحواسيب الأخرى في الثبكة والتي لتي يتوفر فيها منصة Jadex والتطبيق، يقوم الزبون بتنفيذ التطبيق وإدخال أسم الخدمة المراد البحث عنها واسم السلعة التي يرغب في شرائها باختيارها من ضمن السلع المعروضة لديه ويتم ذلك من خلال واجهة المستخدم الخاصة بالزبون. تتم عملية المفاوضات الآلية وسوف تعرض تناصيل باصنيل هذه المفاوضات لدى الزبون والبائع. في حالة انجاز

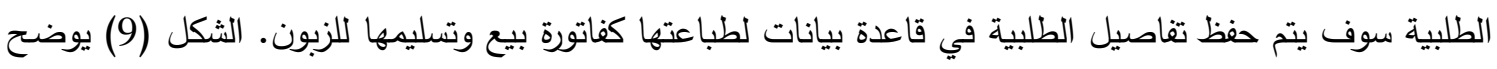
واجهة الوكيل البائع خلال عملية المفاوضات والثكل (10) يوضح واجهة الوكيل المشتري. 


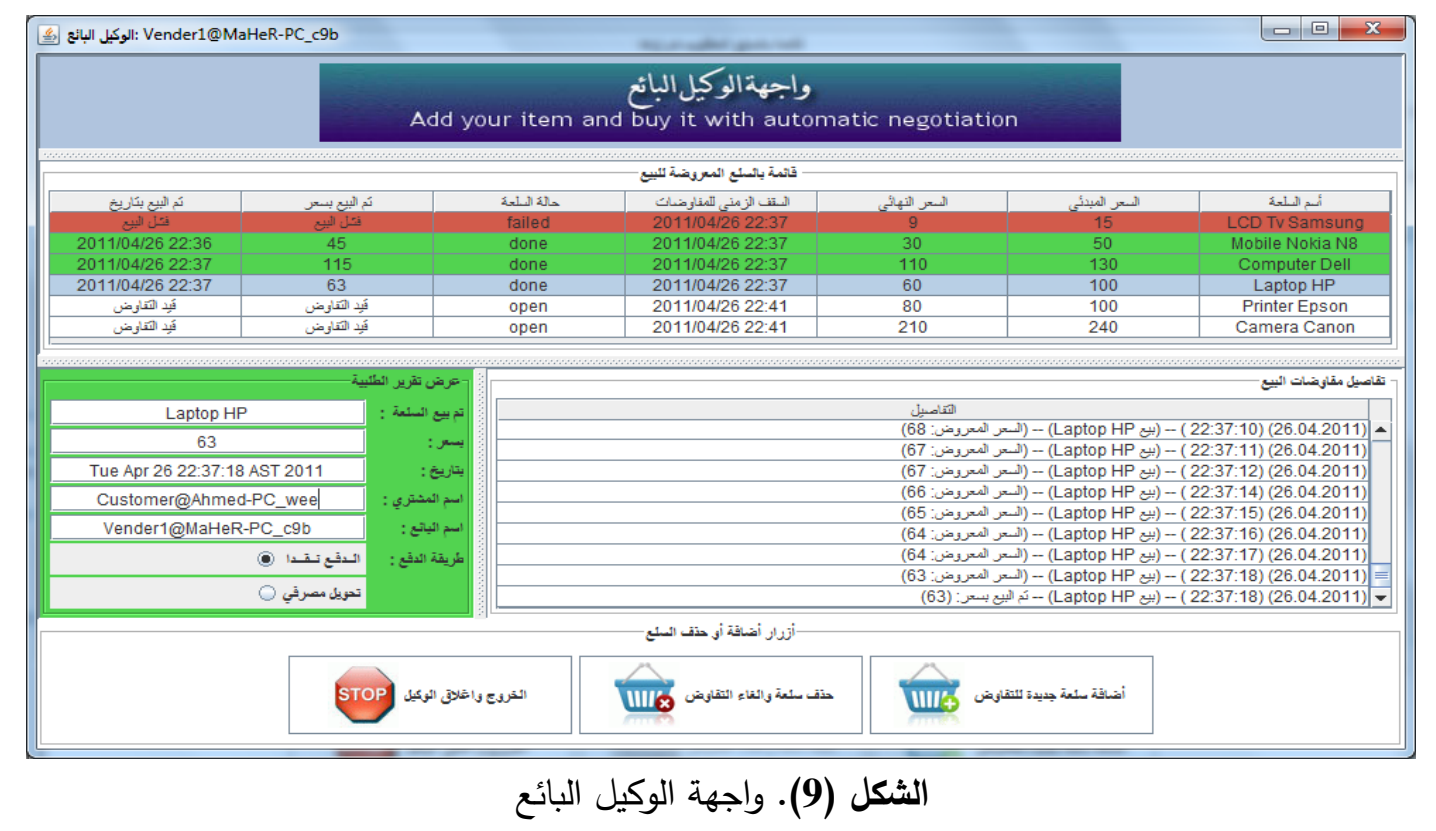

10- الاستنتاجات والأعمال المستقبلية

يهدف الأسلوب المقترح إلى توفير مجموعة من الطرائق والمبادئ التوجيهية لتسهيل عمل مهندسي البرمجيات في عملية تطوير النظم المتعددة الوكلاء، إذ يغطي الأسلوب المقترح أهم مراحل دورة حياة النظام،

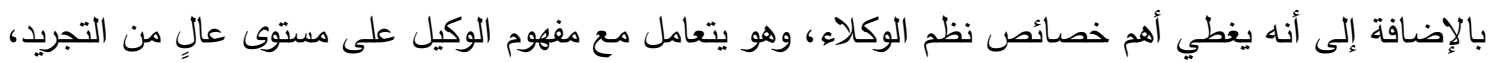

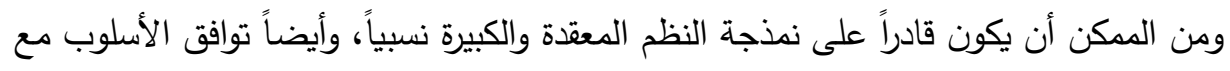

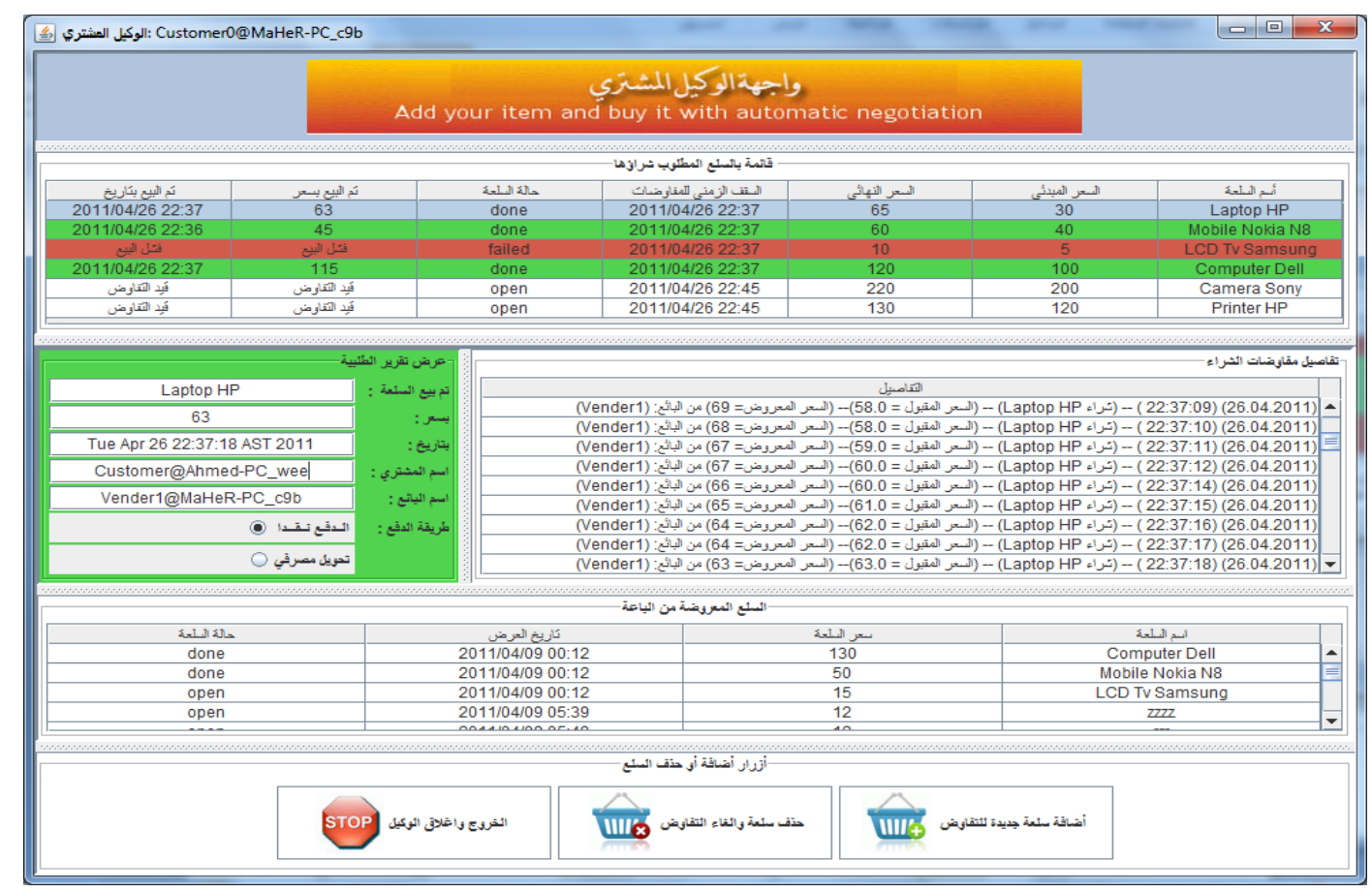

$$
\text { الثكل (10). واجهة الوكيل المشتري }
$$

مقاييس FIPA وهيكلية BDI، ومن خلال عملية تطوير هذا الأسلوب وتجربته من خلال تطبيق التجارة

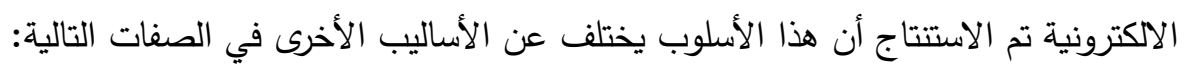


تكامل مراحل التطوير الرئيسية ومن ضمنها المتطلبات المبئية التي تكون في تواصل مباشر مع الزبون. لا توجد إي فجوة بين نماذج التصميم ولغات البرمجة من خلال تفصيل مرحلة البناء التي تستخدم لتحويل نماذج التصميم مباشرة إلى التنفيذ.

تغطية الأسلوب على النماذج المهمة في تطوير الوكلاء مثل نماذج الأدوار والحقائق والأهداف والخطط

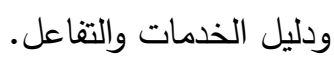
إمكانية تحقيق خصائص الوكلاء مثل الاستقلالية والتعاون والتفاوض والتعلم والاستتتاج والتتقل ولتئل

$$
\text { والخصائص الأخرى. }
$$

$$
\text { السهولة في استخدام أحد بروتوكولات التفاوض وعملية التواصل بين الوكلاء. }
$$

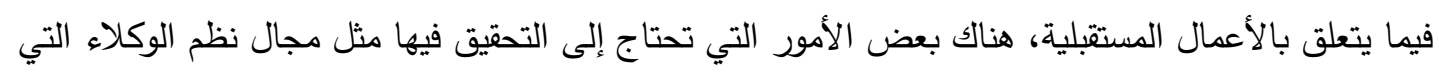

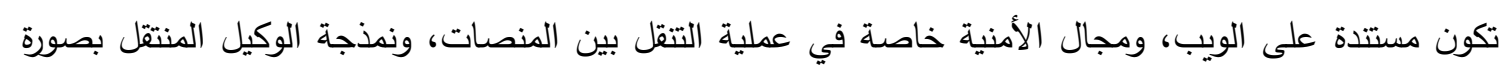
خاصة، ومرحلة فحص التطبيق، وقضايا إدارة المشاريع مثل المقاييس والجدول الزمني والمخاطر والنوعية. 


\section{المصادر}

[1] Abdelaziz, T., Elammari, M., and Branki, C., 2008, "MASD: Towards a Comprehensive Multi-agent System Development Methodology" SpringerVerlag Berlin Heidelberg, pp. 108 - 117.

[2] Al-Hashel, E., Bala M. and Sharma, D., 2007, "A Comparison of Three AgentOriented Software Development Methodologies: ROADMAP, Prometheus, and MaSE" Springer-Verlag Berlin Heidelberg, pp. 909-916.

[3] Basseda, R., Taghiyareh, F., Alinaghi, T., Ghoroghi, C., Moallem, A., 2009, "A Framework for Estimation of Complexity in Agent Oriented Methodologies", IEEE/ACS International Conference on Computer Systems and Applications, aiccsa, pp.645-652.

[4] Bobkowska, A., 2005, "Framework for methodologies of visual modeling language evaluation", Proceedings of the symposia on Meta-informatics, ACM Press, New York.

[5] Bordini, R., Hübner, H., 2005, "Jason", manual, release 0.7, In http://jason.sf.net/.

[6] Braubach, L., Pokahr, A., 2011, "BDI User Guide", In http://jadex-agents.informatik.uni-

hamburg.de/xwiki/bin/view/BDI+User+Guide/03+Agent+Specification

[7] Braubach, L., Pokahr, A., Lamersdorf, W., 2004, "Jadex: A Short Overview", Main Conference Net.ObjectDays, Erfurt, Germany, pp.195-207.

[8] Brazier, F., Dunin, B., Treur, J. and Verbrugge, R., 2001, "Modeling Internal Dynamic Behavior of BDI Agents" Kluwer Academic Publishers, The Hong Kong Institute of Education, Dordrecht, Hong Kong, pp. 339-361.

[9] Bresciani, P., Giorgini, P., Hiunchiglia, F., Mylopoulos, J., Perini, A., 2001, "TROPOS: An Agent-Oriented Software Development Methodology", Technical Report \#DIT-02-0015, AAMAS Journal.

[10] Buhr, R., 1998, "Use Case Maps as Architectural Entities for Complex Systems", IEEE Transactions on Software Engineering 24(12), 1131-1155.

[11] Busetta, P., Ronnquist, R., Hodgson, A. and Lucas, A., 1999, "JACK Intelligent Agents Componenets for Intelligent Agents in Java", updated from AgentLink Newsletter, http://www.agent-software.com.au.

[12] Dam, K., Winikoff, M., 2004, "Comparing Agent-Oriented Methodologies", Giorgini, P., Henderson-Sellers, B., Winikoff, M. (eds.) AOIS 2003. LNCS (LNAI), Springer, Heidelberg, Vol. 3030, pp. 78-93.

[13] Dastani, M., Hulstijn, J., Dignum, F., Meyer, J., 2004, "Issues in Multi-agent System Development", In AAMAS Journal

[14] Finin, T., Labrou, Y., Mayfield, J., 1997, "KQML as an Agent Communication Language" Bradshaw, J. (Ed.) Software Agents. AAAI/MIT Press, pp. 456-463.

[15] FIPA. Foundation for Intelligent Physical Agents, 2002, http://www.fipa.org. 
[16] Foundation for Intelligent Physical Agents, 2002, FIPA Communicative Act Library Specification. Document number SC00037J. Geneva, Switzerland. 45 p.

[17] Foundation for Intelligent Physical Agents, 2002, FIPA Contract Net Interaction Protocol Specification. Document number SC00029H. Geneva, Switzerland. 9p.

[18] JADE. Java Agent Development Framework, 1999, http://jade.cselt.it.

[19] Jeffrey M., 1997, "An introduction to software agents", Jeffrey M. Bradshaw, editor, Software Agents, AAAI Press / The MIT Press, vol. 43,3 p.

[20] Jennings, N. R., Sycara, K. and Wooldridge, M., 1998, "A Roadmap of Agent Research and Development", Autonomous Agents and Multi-Agent Systems vol. 1, pp.275-306.

[21] Jia, Y., Huang, C., Cai, H., 2009, "A Comparison of Three Agent-Oriented Software Development Methodologies: MaSE, Gaia, and Tropos", Springer, Lecture Notes in Computer Science, Vol. 4694, pp. 909-916.

[22] Kolp, M., Castro, J., Mylopoulos, J., 2001, "A Social Organization Perspective on Soft $\neg$ ware Architectures", In Proceedings of the First International Workshop From Software Requirements to Architectures (STRAW 01) at ICSE 2001, Toronto, Canada, pp. 5-12.

[23] Parandoosh, F., 2007, "Evaluating Agent-Oriented Software Engineering Methodologies", IEEE International Workshop on Soft Computing Applications, Gyula, Hungary - Oradea, Romania, pp 21-23.

[24] Rueda, S., Garcia, A. and Simari, G., 2002, "Argument-based Negotiation among BDI Agents," The Journal of Computer Science and Technology (JCS \& T), vol. 2, no. 7, pp.1-8.

[25] Sturm, A., Dori, D., Shehory, O., 2003, "Single-Model Method for Specifying Multi-Agent Systems", The Second International Joint Conference on Autonomous Agents and Multiagent Systems, Melbourne, Australia.

[26] Wood, M., DeLoach, S., 2001, "An Overview of the Multi-agent Systems Engineering Methodology", In Agent-Oriented Software Engineering, P. Ciancarini, M. Wooldridge, (Eds.) Lecture Notes in Computer Science, Springer Verlag, Berlin, vol. 1957, pp.207-222.

[27] Wooldridge, M., Jennings, N. R. and Kinny, D., 2000, "The Gaia Methodology for Agent-Oriented Analysis and Design", Journal of Autonomous Agents and Multi-Agent Systems, 3(3). 